\title{
Design and Mathematical Analysis of a Novel Reluctance Force-Type Hybrid Magnetic Bearing for Flywheel with Gimballing Capability
}

\author{
Chun'e Wang and Jiqiang Tang \\ School of Instrument Science and Opto-electronics Engineering, Beihang University, Beijing 100191, China \\ Correspondence should be addressed to Jiqiang Tang; tjq_72@163.com
}

Received 13 March 2013; Revised 22 April 2013; Accepted 22 April 2013

Academic Editor: Igor Andrianov

Copyright @ 2013 C. Wang and J. Tang. This is an open access article distributed under the Creative Commons Attribution License, which permits unrestricted use, distribution, and reproduction in any medium, provided the original work is properly cited.

\begin{abstract}
Magnetically suspended flywheel (MSFW) with gimballing capability fulfills requirements of precision and maneuvers for space applications. A novel reluctance force-type hybrid magnetic bearing (RFHMB) is presented based on analysis of demerits of Lorentz force-type magnetic bearing and common RFHMB. It features that radial and axial magnetic bearing units are integrated into a compact assembly with four separate biased permanent magnets and two conical stators; four radial poles with shoes and rotor made of iron-based amorphousness can reduce eddy loss. Equivalent magnetic circuits of permanent magnets and their control currents are presented. Simulation results indicate flux density fluctuates from $0.272 \mathrm{~T}$ to $0.41 \mathrm{~T}$; rotor tilting does not affect the suspension force when rotor only tilts around $X$-axis or $Y$-axis. When rotor drifts in $X, Y$, or $Z$ direction and tilts around $X$-axis or $Y$-axis simultaneously, force in corresponding directions slightly increases with tilting angle's enlargement, but the maximum change does not exceed $14 \%$. Additional tilting torque mainly determined by uniformity of flux density in conical air gaps is $0.05 \mathrm{Nm}$ which is far smaller than $11 \mathrm{Nm}$ in common RFHMB; magnetic suspension force is effectively decoupled among $X, Y$, and $Z$ directions; results prove that MSFW with gimballing capability theoretically meets maneuvering requirement of spacecraft.
\end{abstract}

\section{Introduction}

Flywheel is an inertial actuator in a spacecraft attitude control system to generate suitable attitude control torques for correcting spacecraft attitude deviation or adjusting to an assigned attitude [1-4]. Flywheel generates attitude control torque by changing the rotational speed of rotor; this torque has high torque precision but cannot meet the maneuvering requirement fully for some space applications $[5,6]$. Compared with conventional ball bearing flywheels, the magnetically suspended flywheel (MSFW) has advantages of no friction, free lubrication, low losses, and theoretically unlimited lifetime [7-11]. As for the 5-degree-of-freedom (DOF) MSFW, the rotor of it can be actively controlled in all 5 DOFs, so the momentum vector of the flywheel can be tilted actively with respect to the spacecraft body (named as "vernier gimballing") to generate control moment in the other two directions [12,13]. For these space applications of an MSFW with gimballing capability, its structure has been studied in [12, 14]. Bichler and Eckardt [15] and Yabuuchi et al. [16] proposed an attitude control scheme for large angle attitude maneuvers; Kim et al. [14] researched the interaction dynamics between a satellite and on board gimballing MSFWs. Being superior to other attitude actuators, this kind of MSFW promises to fulfill the requirements of both precision and maneuvers and it is possible to decrease the number of the needed flywheels to control the attitude of spacecraft.

With respect to the MSFW with gimballing capability, the magnetic bearings can be divided into Lorentz forcetype magnetic bearings and reluctance force-type magnetic bearings according to the physical cause of the generation of the force. For the Lorentz force-type magnetic bearings, the force per unit length of conductor generated by the interaction of the current $\vec{i}$ and the flux density $\vec{B}$ is orthogonal to the flux lines, independent of the air gap, and linearly dependent on the current [17]. Bichler and Eckardt [15] and Eckardt [18] presented a Lorentz force-type magnetic 
bearing for a low noise momentum wheel with gimballing capability. For the reluctance force-type magnetic bearings, the magnetic force per unit area is given by $B^{2} / \mu_{0}$, where $B$ is the magnetic flux density in the air gap and $\mu_{0}$ is the vacuum permeability. Fang et al. $[19,20]$ and Tang et al. $[19,21]$ paid attention to how to design the structure of a permanent magnet biased axial hybrid magnetic bearing, how to improve its gimballing control ability, and how to reduce its eddy loss when the rotor rotates at a high speed [22]. However, due to the magnetic flux being inversely proportional to the gap length, the tilting angle of rotor is very small and cannot be beyond $0.1^{\circ}$ generally; the generated control moment is only about $0.1 \mathrm{Nms}$, which is too small to meet the maneuvering requirement of spacecraft fully.

In the presented new MSFW with gimballing capability shown in Figure 1, there are one rotor, one novel reluctance force-type hybrid magnetic bearing (RFHMB), one tilting magnetic bearing, one integrated axial/radial displacement sensor, one motor, two touchdown bearings, one shaft, and one base. The presented novel RFHMB, which is located in the center of the flywheel, is used to control both the axial and the radial translation of the rotor. The tilting magnetic bearing shown in Figure 2 is arranged in the outer rim of the flywheel rotor and is used to make the rotor rotate around $X$ - and $Y$-axes by Lorentz force to generate large control moment due to the gyroscope effect of the high-speed rotor. The displacements and tilting angles are measured by the integrated axial/radial displacement sensors. Two touchdown bearings are used to support the rotor when the magnetic bearings are turned off or in case of failure, and allow the rotor spin down from its full operating speed to zero. Due to the novelty and specialty of RFHMB, the rotor of this MSFW with gimballing capability can be tilted beyond $1^{\circ}$ and its outputting control moment is up to $3.3 \mathrm{Nm}$; both of them are larger by far than that of the existing MSFW with gimballing capability. So the presented new MSFW with gimballing capability can meet the maneuvering requirement of spacecraft fully.

This paper emphasizes the design of this novel RFHMB; it features that the radial magnetic bearing unit and axial magnetic bearing unit are integrated as a compact assembly. Permanent magnets are used to generate the radial and axial biased fluxes to support the rotor in order to reduce the consumption of power when this MSFW is on the earth. To improve the suspension properties of this RFHMB, the novel structure of this RFHMB with conical contours for axial suspension is presented firstly to avoid torque coupling when rotor is tilted. In order to decouple the suspension forces not only between $X$ and $Y$ dimension but also between radial direction and axial direction, suspension properties in radial and axial directions, torque characteristics, and coupling properties when rotor is shifted or tilted are researched deeply by theoretical analysis and finite element method. These research results indicate that the additional tilting torque of the bearing can be greatly decreased and the magnetic fluxes among $X, Y$, and $Z$ directions are almost decoupled from each other. The MSFW with gimballing capability can meet the maneuvering requirement of spacecraft theoretically because of the presented novel RFHMB.

\section{Demerits of Existing Reluctance Force-Type Magnetic Bearing for Flywheel with Gimballing Capability}

For the MSFW with gimballing capability, the reluctance force-type magnetic bearings used in it can be classified into two types generally according to their arrangement and structure. One type shown in Figure 3 includes an upper 2DOF radial magnetic bearing (2-DOF $\left.\mathrm{RMB}_{-\mathrm{UP}}\right)$, a lower 2DOF radial magnetic bearing (2-DOF $\mathrm{RMB}_{-\mathrm{LO}}$ ), an upper 1DOF axial magnetic bearing (1-DOF $\left.\mathrm{AMB}_{-\mathrm{UP}}\right)$, and a lower $1-\mathrm{DOF}$ axial magnetic bearing (1-DOF $\left.\mathrm{AMB}_{-\mathrm{LO}}\right)$. The structures and photos of 2-DOF RMB and 1-DOF AMB are shown in Figure 4, respectively. Another type including one 2-DOF RAM, an upper 3-DOF axial magnetic bearing (3-DOF $\mathrm{AMB}_{-\mathrm{UP}}$ ), and a lower 3-DOF axial magnetic bearing (3$\mathrm{DOF}_{A M B} \mathrm{AO}_{-\mathrm{O}}$ ) is shown in Figure 5, the structure of 2-DOF $\mathrm{RMB}$ is the same as that in Figure 4(b), and the structures and photos of 3-DOF AMB are shown in Figure 6 [21]. All of the 2-DOF RMB, 1-DOF AMB, and 3-DOF AMB are permanent magnet biased magnetic bearings; having positive displacement stiffness is one of their intrinsic characteristics.

For the MSFW shown in Figure 3 , set $J_{p}, J_{e}$, and $\Omega$ as the polar inertial moment, equatorial inertial moment, and the rotational speed of rotor, respectively. If we want to tilt the rotor around $X$-axis (or $Y$-axis) anticlockwise for angle $\varphi$ per unit time by this pair of 2-DOF RMBs to generate the required gyroscope momentum $M_{\text {req }}$, then

$$
M_{\text {req }}=J_{p} \Omega \times \dot{\varphi}
$$

The generation of additional tilting torque is shown in Figure 7, where $L_{r}$ presents the distance between the center of 2 -DOF $\mathrm{RMB}_{\text {-UP }}$ or 2-DOF $\mathrm{RMB}_{\text {-LO }}$ and $Y$-axis. If we denote $F_{\text {ru-l }}$ and $F_{\text {ru-r }}$ as the left suction force and the right suction force of 2-DOF $\mathrm{RMB}_{-\mathrm{UP}}$, respectively, their unbalanced force is $F_{\text {ru-r }}-F_{\text {ru-1 }}$. In the same manner, we denote $F_{\text {rl-1 }}$ and $F_{\text {rl-r }}$ as the left suction force and right suction force of 2-DOF $\mathrm{RMB}_{\text {-LO }}$, their unbalanced force with being $F_{\mathrm{rl}-\mathrm{l}}-F_{\mathrm{rl}-\mathrm{r}}$, then the required driving momentum $M_{d}$ is

$$
M_{d}=J_{e} \cdot \dot{\varphi}=\left(F_{\text {ru-r }}-F_{\text {ru-l }}+F_{\text {rl-1 }}-F_{\text {rl-r }}\right) \cdot L_{r} .
$$

Then (1) becomes

$$
M_{\text {req }}=J_{p} \Omega \times\left[\frac{F_{\text {ru-r }}-F_{\text {ru-l }}+F_{\mathrm{rl}-1}-F_{\mathrm{rl}-\mathrm{r}}}{J_{e}} \cdot L_{r}\right] .
$$

However due to the tilting angle, the right suction force of $1-\mathrm{DOF} \mathrm{AMB}_{\text {-UP }}$ presented by $F_{\text {au-r }}$ becomes larger than its left suction force $F_{\text {au-l }}$, and the left suction force of 1-DOF $\mathrm{AMB}_{\text {-LO }}$ presented by $F_{\mathrm{al}-\mathrm{l}}$ becomes larger than its right suction force $F_{\mathrm{al}-\mathrm{r}}$. If the distance between the force center of 1-DOF $\mathrm{AMB}_{-\mathrm{UP}}$ or 1-DOF $\mathrm{AMB}_{-\mathrm{LO}}$ and $Z$-axis is $L_{a}$, then the additional torque $M_{a}$ acting on the rotor produced by 1DOF AMBs is

$$
M_{a}=\left(F_{\mathrm{au}-\mathrm{r}}-F_{\mathrm{au}-1}+F_{\mathrm{al}-1}-F_{\mathrm{al}-\mathrm{r}}\right) \cdot L_{a} \cdot
$$




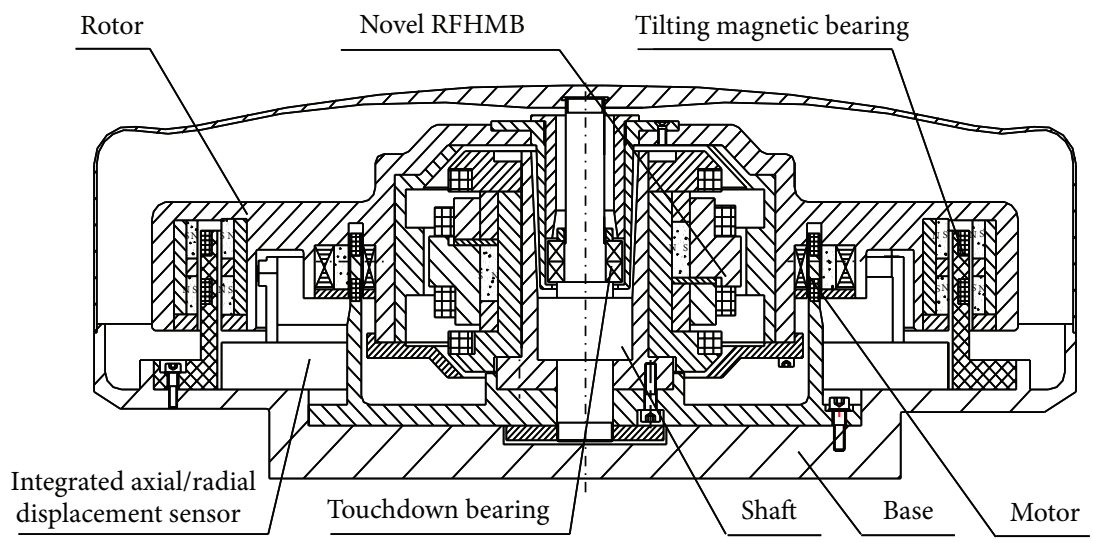

FIGURE 1: Momentum flywheel with gimballing capability.

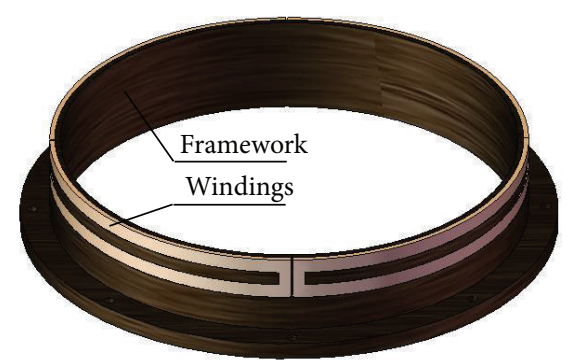

FIGURE 2: Stator of Lorentz force-type tilting magnetic bearing.

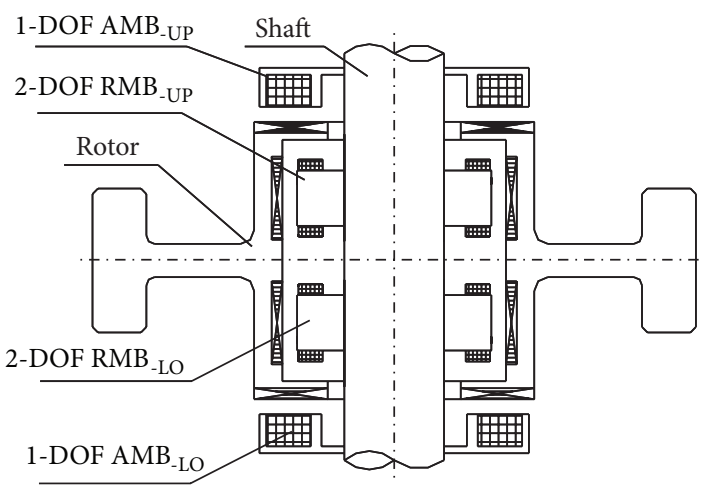

FIGURE 3: MSFW with 2-DOF RMBs and 1-DOF AMBs.

So the actual outputting gyroscope momentum $M_{\text {act }}$ of the rotor is

$$
\begin{aligned}
M_{\mathrm{act}}=J_{p} \Omega \times[ & \left(F_{\mathrm{ru}-\mathrm{r}}-F_{\mathrm{ru}-\mathrm{l}}+F_{\mathrm{rl}-\mathrm{l}}-F_{\mathrm{rl}-\mathrm{r}}\right) \cdot L_{r} \\
& \left.+\left(F_{\mathrm{au}-\mathrm{r}}-F_{\mathrm{au}-1}+F_{\mathrm{al}-\mathrm{l}}-F_{\mathrm{al}-\mathrm{r}}\right) \cdot L_{a}\right] \times\left(J_{e}\right)^{-1} .
\end{aligned}
$$

The error between the required gyroscope momentum $M_{\text {req }}$ and the actual outputting gyroscope momentum $M_{\text {act }}$ is $J_{p} \Omega \times\left[\left(F_{\mathrm{au}-\mathrm{r}}-F_{\mathrm{au}-1}+F_{\mathrm{al}-1}-F_{\mathrm{al}-\mathrm{r}}\right) \cdot L_{a}\right] / J_{e}$, which is difficult to be measured or be compensated effectively, so the attitude of the spacecraft cannot be controlled accurately.
Similarly, if the rotor is tilted around $X$-axis (or $Y$-axis) anticlockwise for angle $\varphi$ per unit time by outer torque, which maybe produced by some special mechanism such as the tilting magnetic bearings shown in Figures 1 and 2 to generate the required gyroscope momentum, the error between the required gyroscope momentum $M_{\text {req }}$ and the actual outputting gyroscope momentum $M_{\text {act }}$ is $J_{p} \Omega \times$ $\left[\left(F_{\mathrm{ru}-\mathrm{r}}-F_{\mathrm{ru}-\mathrm{l}}+F_{\mathrm{rl}-\mathrm{l}}-F_{\mathrm{rl}-\mathrm{r}}\right) \cdot L_{r}+\left(F_{\mathrm{au}-\mathrm{r}}-F_{\mathrm{au}-\mathrm{l}}+F_{\mathrm{al}-\mathrm{l}}-F_{\mathrm{al}-\mathrm{r}}\right) \cdot L_{a}\right] / J_{e}$, which is larger than that produced by a pair of 2-DOF RMBs.

For the MSFW shown in Figure 5, the generation of additional tilting torque is shown in Figure 8. Setting the distance between the force center of 3-DOF $\mathrm{AMB}_{-\mathrm{UP}}$ or 3DOF $\mathrm{AMB}_{\text {-LO }}$ and $Z$-axis as $L_{a}$, when the rotor is tilted around $X$-axis (or $Y$-axis) anticlockwise for angle $\varphi$ per unit time by this pair of 3-DOF AMBs to generate the required gyroscope momentum $M_{\text {req }}$, the required driving momentum $M_{d}$ provided the left suction force and the right suction force of 3-DOF AMB -UP $_{\text {p }}$ presented by $F_{\mathrm{au}-1}$ and $F_{\mathrm{au}-\mathrm{r}}$; the left suction force and the right suction force of 3-DOF $\mathrm{AMB}_{\text {-LO }}$ presented by $F_{\mathrm{al}-\mathrm{l}}$ and $F_{\mathrm{al}-\mathrm{r}}$ are

$$
M_{d}=J_{e} \cdot \dot{\varphi}=\left(F_{\mathrm{au}-\mathrm{r}}-F_{\mathrm{au}-\mathrm{l}}+F_{\mathrm{al}-\mathrm{l}}-F_{\mathrm{al}-\mathrm{r}}\right) \cdot L_{a} .
$$

For the 2-DOF RMB in this MSFW, the left suction force presented by $F_{\mathrm{r}-1}$ equals the right suction force presented by $F_{r-r}$, and both of them cross through the centroid of rotor $O$, and then the torque made by them is zero and the actual outputting gyroscope momentum $M_{\text {act }}$ of the rotor is not affected. But the tilting angle of rotor is very small and cannot be beyond $0.1^{\circ}$ generally because the air gaps of both 2-DOF RAM and 3-DOF AMB are about $0.3 \mathrm{~mm}$, which is too small for the rotor to tilt about a large angle.

If the rotor is tilted around $X$-axis (or $Y$-axis) anticlockwise for angle $\varphi$ per unit time by outer torque produced by tilting magnetic bearings shown in Figures 1 and 2 to generate the required gyroscope momentum, the torque presented by (6) becomes the additional torque acting on the rotor, so the error between the required gyroscope momentum $M_{\text {req }}$ and the actual outputting gyroscope momentum $M_{\text {act }}$ is $J_{p} \Omega \times$ $\left(F_{\mathrm{au}-\mathrm{r}}-F_{\mathrm{au}-\mathrm{l}}+F_{\mathrm{al}-\mathrm{l}}-F_{\mathrm{al}-\mathrm{r}}\right) \cdot L_{a} / J_{e}$, which is also difficult to be measured or be compensated effectively, so the attitude of the spacecraft cannot be controlled accurately either. 

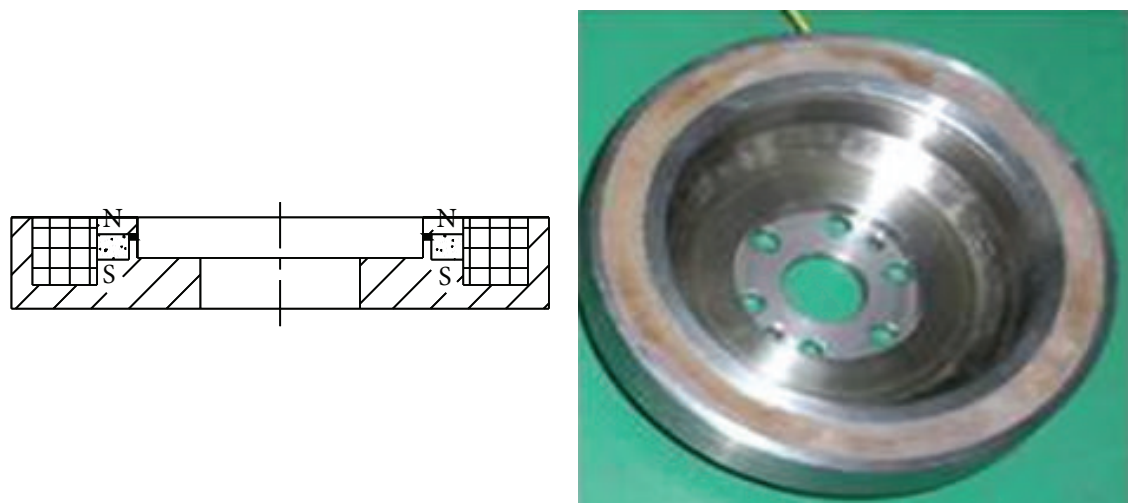

(a)
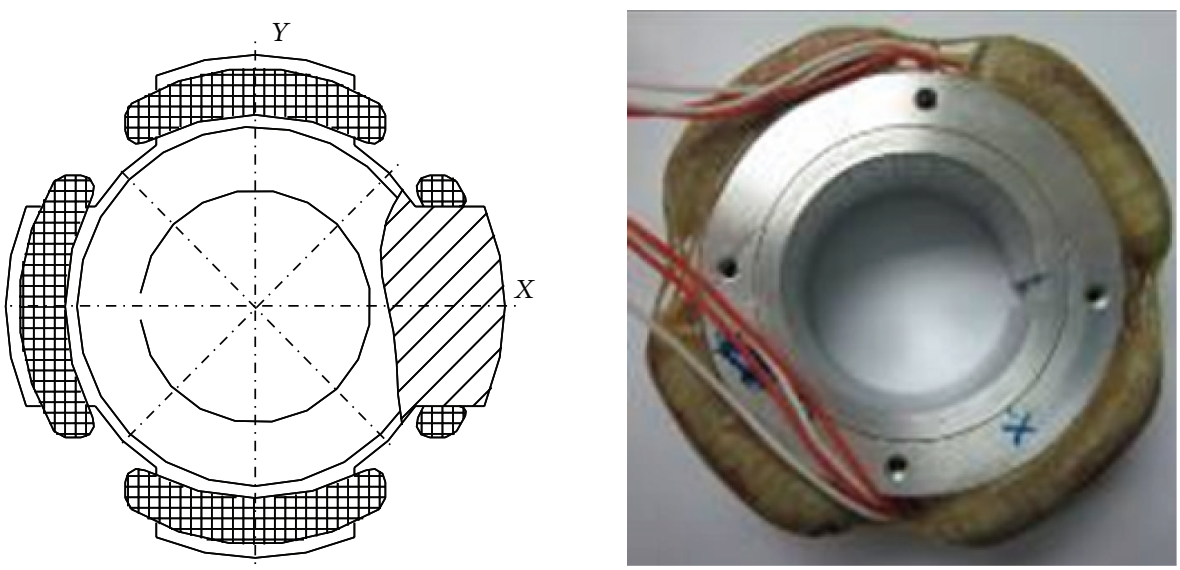

(b)

FIGURE 4: Structure and photos of magnetic bearings, (a) 1-DOF AMBs, (b) 2-DOF RMBs.

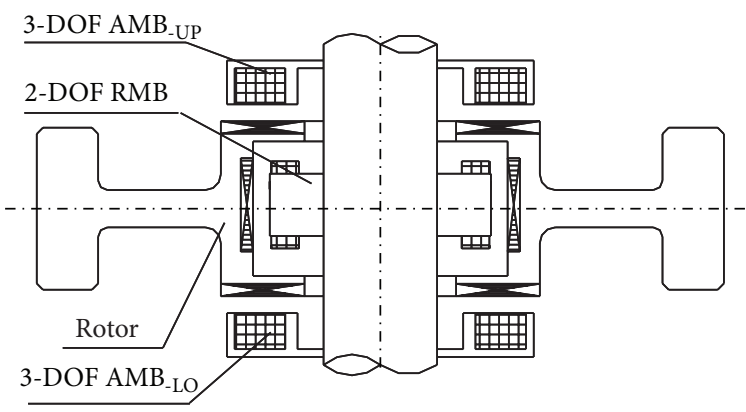

FIgURE 5: MSFW with one 2-DOF RAM and two 3-DOF AMBs.

However, no matter what the additional torque is, the additional torque acting on the rotor is equal to the product of the unbalanced force and torque arm of the bearing. Even if the positive displacement stiffness is the intrinsic characteristic of $\mathrm{AMB}$ or $\mathrm{RMB}$ and it is difficult to reduce these unbalanced forces when the rotor tilts, it is preferable to reduce the torque arm of $\mathrm{AMB}$ or $\mathrm{RMB}$ by adopting the novel RFHMB to make the additional torque to be zero approximately.

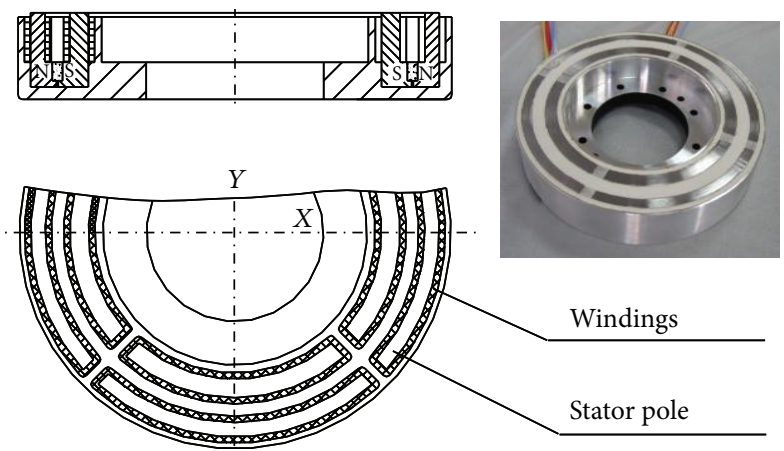

Figure 6: Structure and photos of 3-DOF AMB.

\section{Structure Design and Magnetic Analysis}

3.1. Structure. Yabuuchi et al. [16], Sawada et al. [13], and Horiuchi and Inoue [23] presented a conical pure active reluctance force-type magnetic bearing for the attitude control of satellite. When these kinds of magnetic bearings are used in MSFW with gimballing capability, the air gap of them are larger than those in common magnetic bearings to enlarge the tilt angle of the flywheel rotor, but they will consume plenty of power to support the rotor on the earth. Since 


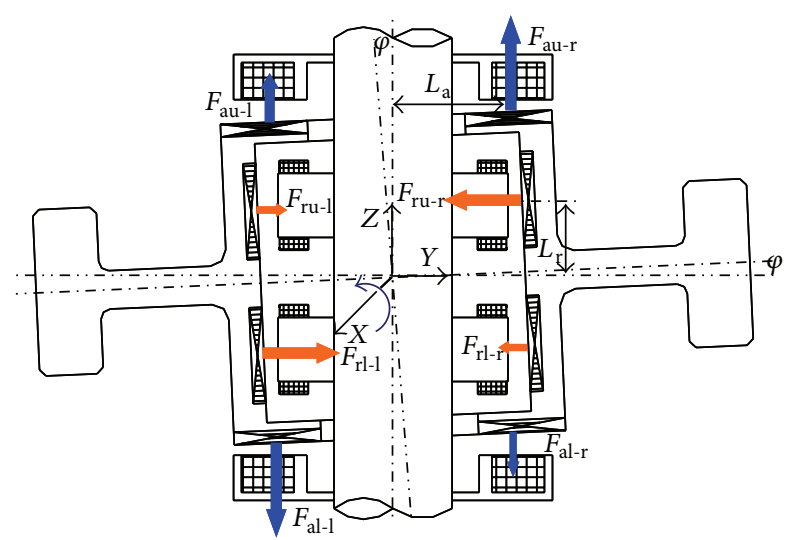

FIGURE 7: Generation of additional tilting torque on rotor for MSFW with 2-DOF RMBs and 1-DOF AMBs during tilting.

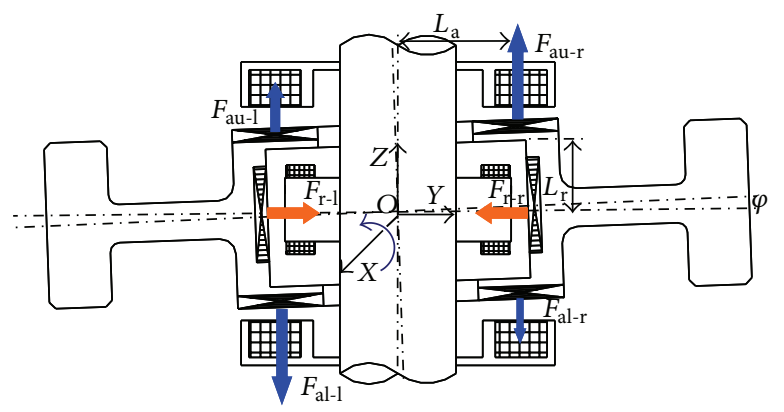

FIGURE 8: Generation of additional tilting torque on rotor for MSFW with 2-DOF RMBs and 1-DOF AMBs during tilting.

the force generated by conical stator has component forces in both radial and axial directions, it is crucial to solve the problem of force decoupling to make its control easier. When the flywheel rotor tilts with respect to the stator, the air gap is not uniform any more and the flux in one channel (e.g., $X$ channel) is prone to leak into adjacent channel ( $Y$ channel), which will make the coupling between different channels more serious. In order to solve the problem of coupling between radial and axial forces in conical magnetic bearing, Watkins et al. proposed a structure with pole piece separated into two parts tapering mirror images of one another [24], but this structure will result in large additional tilting torque when it is used in MSFW with gimballing capability.

For this presented novel RFHMB as shown in Figure 9, its main function is to ensure that the rotor of this MSFW be suspended stably in the central position, so the special structure of RFHMB is our original novelty and how to analyze its suspension properties is our main contributions. In this novel RFHMB, the radial magnetic bearing unit and axial magnetic bearing unit are integrated as a hybrid reluctance force-type magnetic bearing assembly. Permanent magnets are used to generate the radial and axial bias fluxes to suspend the rotor without consumption lots of power when this MSFW is on the earth. The radial stators shown in Figure 10 include 4 stator poles and are located in the center of RFHMB, their relative permanent magnets are also separated into four separate segments by nonmagnetic materials among them. Two stator poles in the same channel are connected by the stator yoke to be a stator subassembly in $X$ or $Y$ channels. These radial stator subassemblies of $X$ and $Y$ channels are connected perpendicularly with nonmagnetic material between them, so the magnetic flux of $X$ and $Y$ channels are independent from each other. There are two conical stators, one is located at the upper end and another is located at the lower end, and each axial conical stator has one control winding. These conical stators are used to control the translation of rotor in $Z$ direction only and have no effects on these forces in radial direction. Because these two conical working air gaps formed between the stator and rotor of the bearing can make the normal direction of the midst conical faces direct to the centroid of the rotor, so the torque arm of the bearing is approximately to zero, the additional torque acting on the rotor can be reduced approximately to zero consequently when the rotor is tilted.

3.2. Magnetic Analysis. The rotor of the MSFW with gimballing capability is suspended in the central position by the novel RFHMB. When the rotor is disturbed to move to $Z+$ direction, the upper conical air gap would become larger while the lower conical air gap would get smaller along $Z$ direction, so the flux density and force would get even smaller at the upper conical stator, and the rotor would subject to an additional force in $Z+$ direction. The integrated axial/radial displacement sensor can detect this axial translation of rotor and transmit related signals to the control system to prevent the rotor from translating further in $Z+$ direction. Then the control system sends switching signals to generate control current through these axial windings; this process results in a suitable corrective control flux. The novel RFHMB with flux paths in $X$ direction is shown in Figure 11, where the biased fluxes generated by permanent magnets are denoted by the solid lines and the control flux generated by windings are denoted by dash line. The biased flux generated from the permanent magnet, which is radically magnetized, is divided into two parts after passing through the radial air gaps: one is the biased flux flowing across the radial stator pole, air gap, the rotor, upper axial air gap, and its upper axial stator; the other is a flux flowing across the radial stator pole, the air gap, the rotor, the lower axial air gap, and its lower axial stator. The control flux generated by the current in axial controlling windings flows across the upper stator pole, the upper axial air gap, the rotor, the lower axial air gap, and the lower stator pole. By adding the biased flux at the large air gap side and subtracting the biased flux at the small air gap side, the bearing can produce a restoring force along the axial direction.

When the rotor moves in $X+$ direction, the flux density and force under the $X+$ radial stator pole decrease while those under the $X$ - radial stator pole increase, so an additional force is generated in $X+$ direction. Due to the conical stators in axial magnetic bearing, their flux density and force would also decrease under the $X+$ part. Since the component forces in $Z$ direction generated by the upper and lower conical stators can be counteracted with each other, the component 


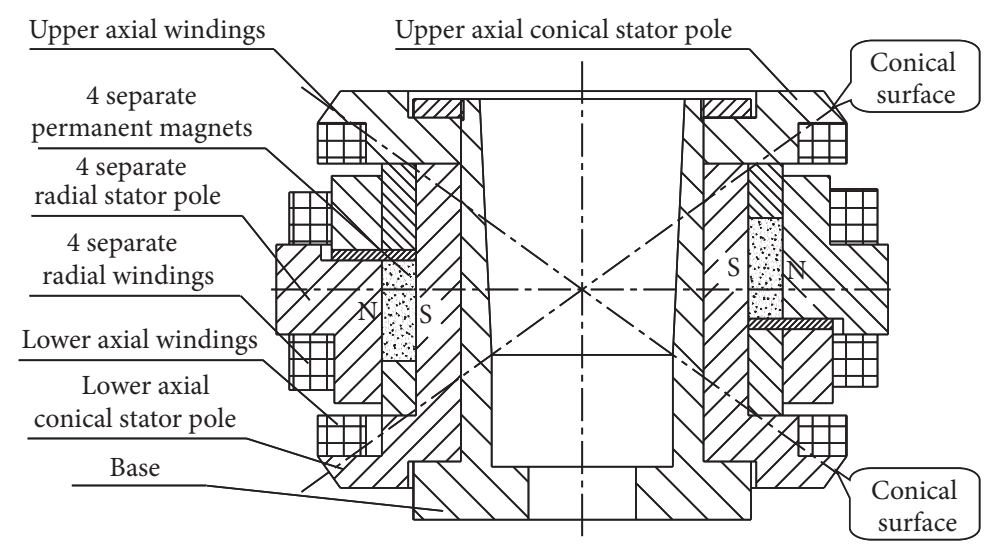

Figure 9: Schematic diagram of novel RFHMB.

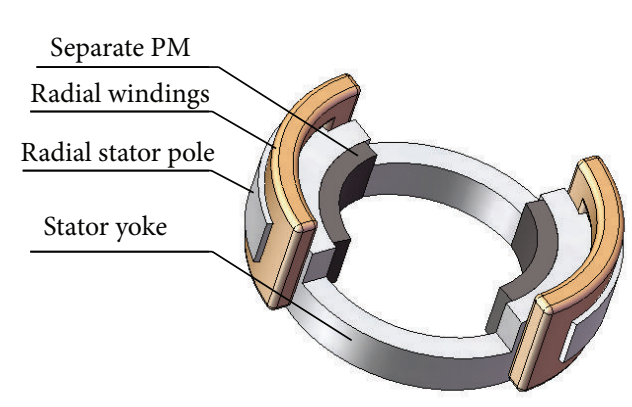

(a)

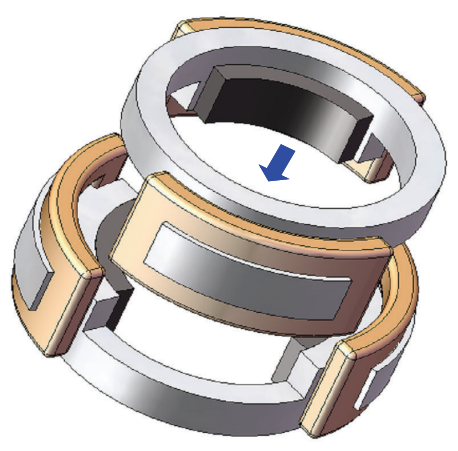

(b)

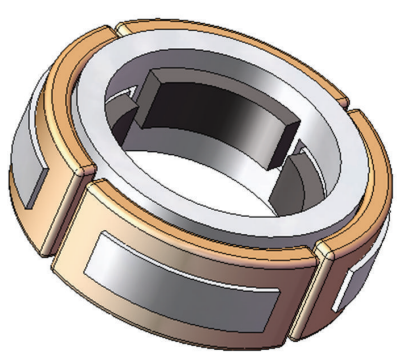

(c)

Figure 10: Radial stator of the novel RFHMB, (a) one stator subassembly in $X$ or $Y$ channels, (b) combination of two stator assemblies, (c) radial stator.

force in $X$ direction is only left. The integrated axial/radial displacement sensor can also detect this state and transmit related signals to the control system to prevent the rotor from translating further in $X+$ direction. The control flux generated by the control current in radial windings in $X$ channel is represented by dash line in Figure 12; it flows through the $X+$ radial air gap, the $X$ channel stator yoke, and the $\mathrm{X}$ - radial air gap; then the bias flux in the $X+$ direction is enhanced and the biased fluxes in the $X$ - direction is decreased; therefore, a restoring force is produced and the rotor is moved back toward the central position.

If we define $F_{\mathrm{pm}}$ and $R_{\mathrm{pm}}$ as the magnetic motive force and reluctance of each permanent magnet $(A), \delta_{x}, \delta_{y}$, and $\delta_{z}$ as the displacement in $X, Y$, and $Z$ directions (m), respectively, the reluctance of radial air gap in radial magnetic bearing unit in $X+$ direction is presented by $R_{x+}(1 / \mathrm{H})$, that in $X-$ direction is presented by $R_{x-}(1 / \mathrm{H})$. Similarly, $R_{y+}$ and $R_{y-}$ present reluctance of radial air gap in $Y+$ and $Y$-direction for the radial magnetic bearing unit $(1 / \mathrm{H}) ; R_{z+}$ and $R_{z-}$ present reluctance of air gap of axial magnetic bearing unit in $Z+$ and $Z$ - direction $(1 / H)$, respectively. At the same time, the control currents in $X$ direction and $Y$ direction for radial windings and that for axial windings are presented by $I_{x}$,
$I_{y}$, and $I_{z}(A)$; their relative magnetic motive forces of each control windings are presented by $F_{c x}(A), F_{c y}(A)$, and $F_{c z}(A)$, respectively. $F_{c x}=N_{r} I_{x}, F_{c y}=N_{r} I_{y}$, and $F_{c z}=N_{a} I_{z}$, where $N_{r}$ is the turn number of the radial control windings and $N_{a}$ is the turn number of the axial control windings.

Considering the influence of the leaked flux, the equivalent magnetic circuits of permanent magnets in this novel RFHMB are simplified as shown in Figure 13, and its equivalent magnetic circuits of control current are simplified as shown in Figure 14. From Figures 13 and 14, we can find out that not only the magnetic fluxes of the radial magnetic bearing unit in $X$ and $Y$ channels are independent from each other, but also the magnetic fluxes of these conical stators have no effect on that of the radial magnetic bearing unit. Even if the air gap becomes uniform badly when the flywheel rotor tilts with respect to the stator, there are no couple between $X$ and $Y$ channels in the radial magnetic bearing unit or between radial magnetic bearing unit and axial magnetic bearing unit at all. So the difficult problem of decoupling between different channels is solved by this presented special structure accordingly.

According to the equivalent magnetic circuit shown in Figures 13 and 14, the biased fluxes and control fluxes of each 


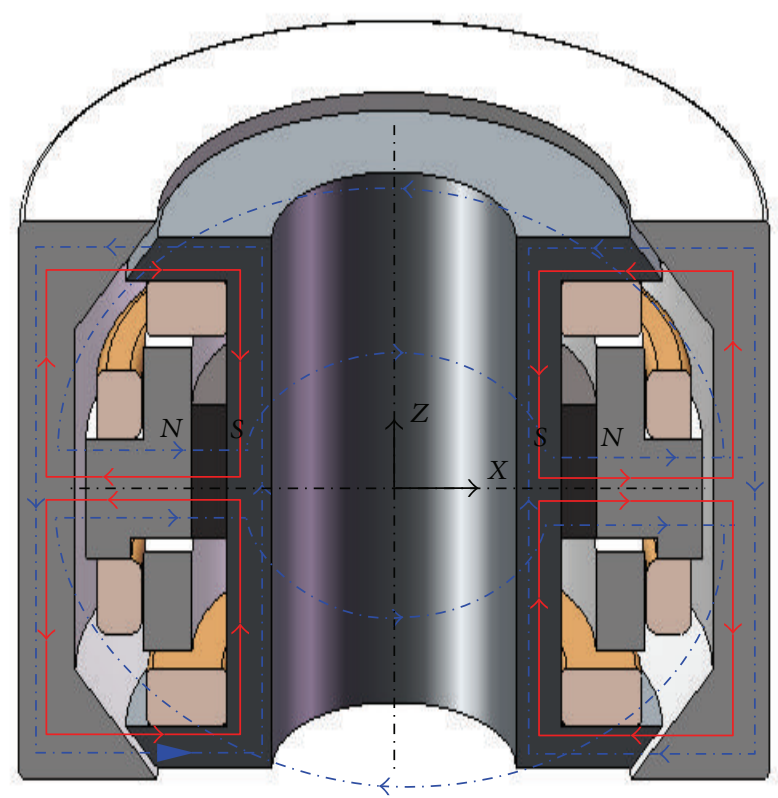

FIGURE 11: Novel RFHMB with paths of flux in $X$ direction.

air gaps can be calculated out according to the Ohm's Law of the magnetic circuit:

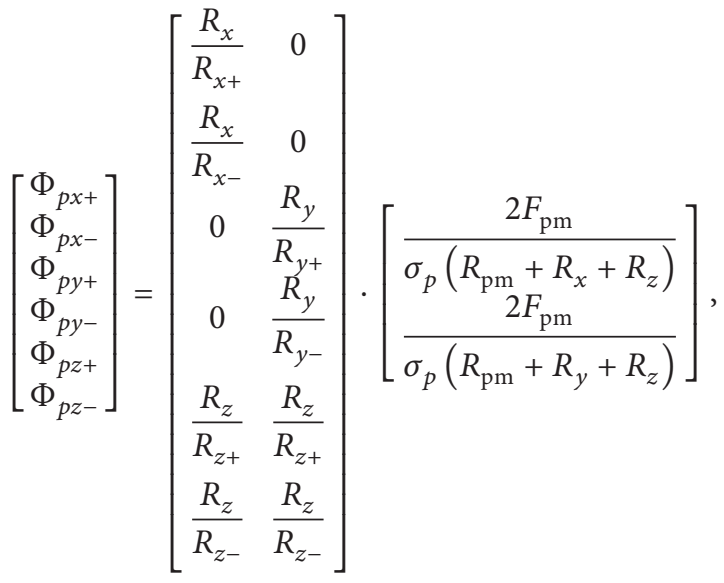

where $\sigma_{p}$ is the leakage coefficient of the biased flux and $\sigma_{r}$ and $\sigma_{z}$ are the leakage coefficients of the radial and axial control fluxes, respectively. $R_{x}=1 /\left(1 / R_{x+}+1 / R_{x-}\right), R_{y}=$ $1 /\left(1 / R_{y+}+1 / R_{y-}\right)$, and $R_{z}=1 /\left(1 / R_{z+}+1 / R_{z-}\right)$.

And the control flux in each channel can be expressed as

$$
\begin{aligned}
& \Phi_{c x}=\frac{2 F_{c x}}{\left[\left(R_{x+}+R_{x-}\right) \cdot \sigma_{r}\right]}, \\
& \Phi_{c y}=\frac{2 F_{c y}}{\left[\left(R_{y+}+R_{y-}\right) \cdot \sigma_{r}\right]}, \\
& \Phi_{c z}=\frac{2 F_{c z}}{\left[\left(R_{z+}+R_{z-}\right) \cdot \sigma_{z}\right]} .
\end{aligned}
$$

Hence the total flux of each air gap can be expressed as

$$
\begin{array}{ll}
\Phi_{x+}=\Phi_{p x+}+\Phi_{c x}, & \Phi_{x-}=\Phi_{p x-}-\Phi_{c x}, \\
\Phi_{y+}=\Phi_{p y+}+\Phi_{c y}, & \Phi_{y-}=\Phi_{p y-}-\Phi_{c y}, \\
\Phi_{z+}=\Phi_{p z+}+\Phi_{c z}, & \Phi_{z-}=\Phi_{p z^{-}}-\Phi_{c z},
\end{array}
$$

where $\Phi_{x+}$ is the total flux of bias and control fluxes in $X+$ direction, $\Phi_{x_{-}}$is the total flux in $X$ - direction, $\Phi_{y+}$ and $\Phi_{y-}$ are the total fluxes in $Y+$ and $Y$ - directions, respectively, and $\Phi_{z+}$ and $\Phi_{z_{-}}$are the total fluxes in $Z+$ and $Z$ - directions, respectively.

In order to express the magnetic field distribution of the novel RFHMB explicitly, the 3D FEM model of the designed RFHMB is set up in ANSYS 11.0. When the rotor locates at the equilibrium position, the static biased magnetic field distribution of the bearing is obtained as shown in Figure 15.

\section{Analysis of Eddy Loss}

In the novel RFHMB, the segmented radial stator poles will make the magnetic density in the radial rotor uneven unavoidably; what will result in the enormous eddy loss when the rotor rotates at high speed. The power consumption of the RFHMB includes the following: (1) copper loss $P_{\mathrm{cu}}$ caused by the windings in RFHMB, and $P_{\mathrm{cu}}=i^{2} R$, where $i$ is the current in the winding and $R$ is the resistant of the winding; (2) iron loss is caused by the magnetic field, which is produced not only by the alternative modulating current in windings but also by the biased permanent magnets. Iron loss includes static suspension iron loss and rotational iron loss and can also be divided into hysteresis loss $P_{h}$ and eddy loss $P_{e}$ :

$$
\begin{gathered}
P_{h}=K_{h} * f_{h} * B_{m}^{1.6} V_{f e}, \\
P_{e}=K_{e} \Delta^{2} f_{h}^{2} B_{m}^{2} V_{f e},
\end{gathered}
$$

where $K_{h}$ is the material constant, $K_{e}$ is coefficient of the material constant, $f_{h}$ is the alternative frequency of the magnetic field produced by the alternative current, $B_{m}$ is the magnitude of the changed magnetic density for the alternating magnetic field, $\Delta$ is the thickness of the material, and $V_{f e}$ is the volume of the iron core for the magnetic pole [21].

With respect to the RFHMB, there is a biased magnetic field and free of magnetic field alternation between positive and negative, so the hysteresis loss $P_{h}$ is small enough to be neglected. The eddy loss $P_{e}$ is an important issue to be focused on. According to (11), we can reduce the eddy loss of the novel RFHMB by optimization of stator poles to reduce the magnitude of the changed magnetic density for the alternating magnetic field and selection of the soft magnetic material with low loss properties such as laminated material, iron-based Nanocrystalline, and amorphousness.

When the stator pole of RFHMB is divided into four segments, a qualitative conclusion can be drawn that the magnetic density in the radial rotor plate produced by these poles would change when the rotor rotates. In order to make the difference of the magnetic density affected by the permanent 


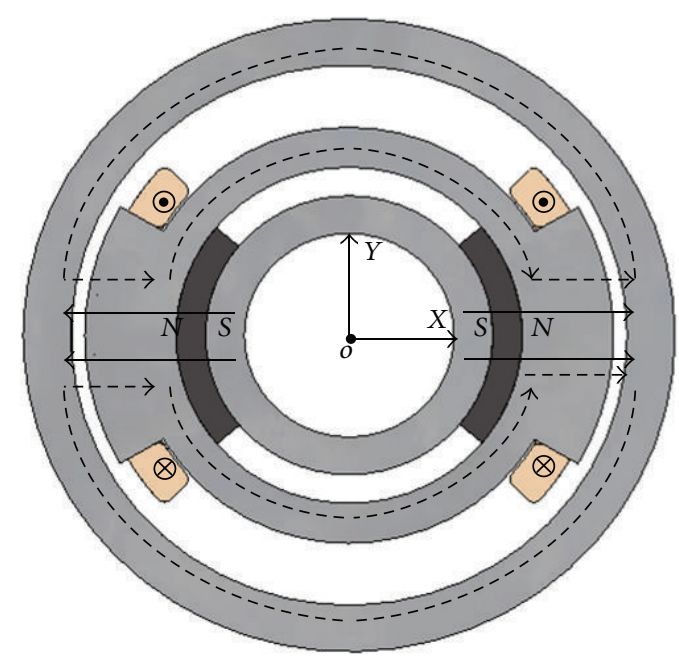

FIGURE 12: Paths of radial flux in $X$ direction for the novel RFHMB.

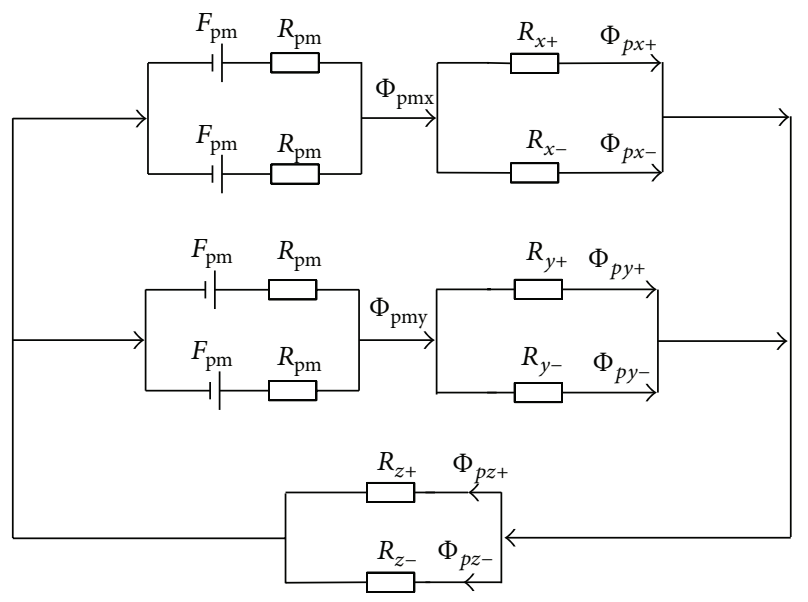

FIGURE 13: Equivalent magnetic circuits of permanent magnets in the RFHMB.
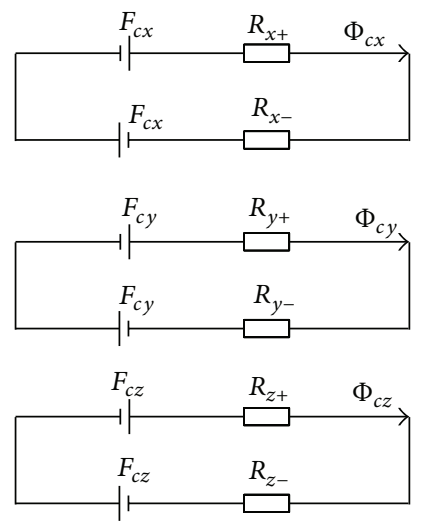

FIGURE 14: Equivalent magnetic circuits of control current.
1

Nodal solution

Step $=2$

Sub $=1$

Time $=2$

BSUM (avg)

RSYS $=14$

$\mathrm{SMN}=0.716 E-05$

SMX $=2.051$
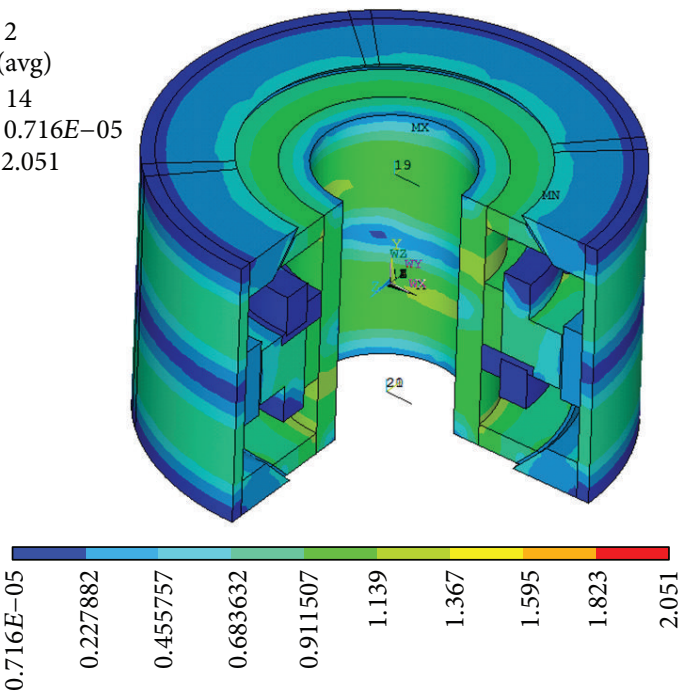

FIGURE 15: Magnetic field distribution of the RFHMB.

magnets and/or the control current in the air gap smaller, we designed a special structure of the stator pole with shoes for the novel RFHMB. The original stator poles without shoes are shown in Figures 16(a); 16(b) is the diagram of stator with poles shoes. From Figure 16, we can find out that the clearance between poles has been reduced from $30^{\circ}$ to $5^{\circ}$. In order to satisfy the requirement of high magnet energy product and small temperature coefficient, these separate permanent magnets in the RFHMB are samarium cobalt permanent magnet and their hysteresis force $H_{\mathrm{cb}}$ and remanence $B_{r}$ are larger than $760 \mathrm{KA} / \mathrm{m}$ and $1.05 \mathrm{~T}$, respectively. When the air gap $\delta$ of the RFHMB is $0.25 \mathrm{~mm}$, the radial distributions of magnetic density affected by only the permanent magnets or 


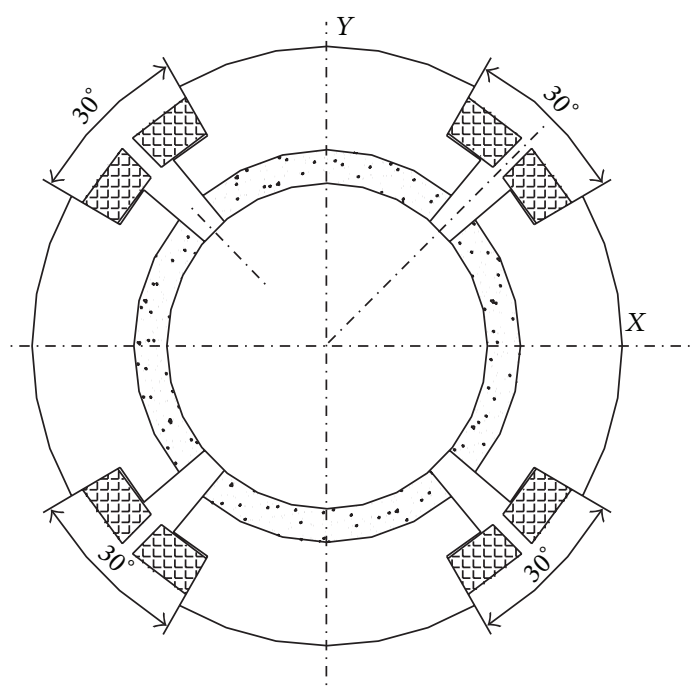

(a)

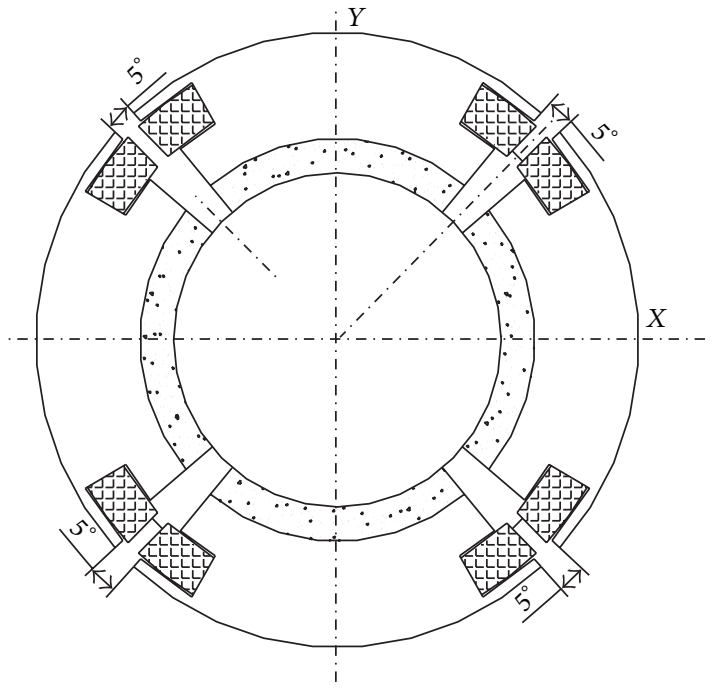

(b)

FIGURE 16: Radial stator of the novel RFHMB, (a) without pole shoes, (b) with pole shoes.

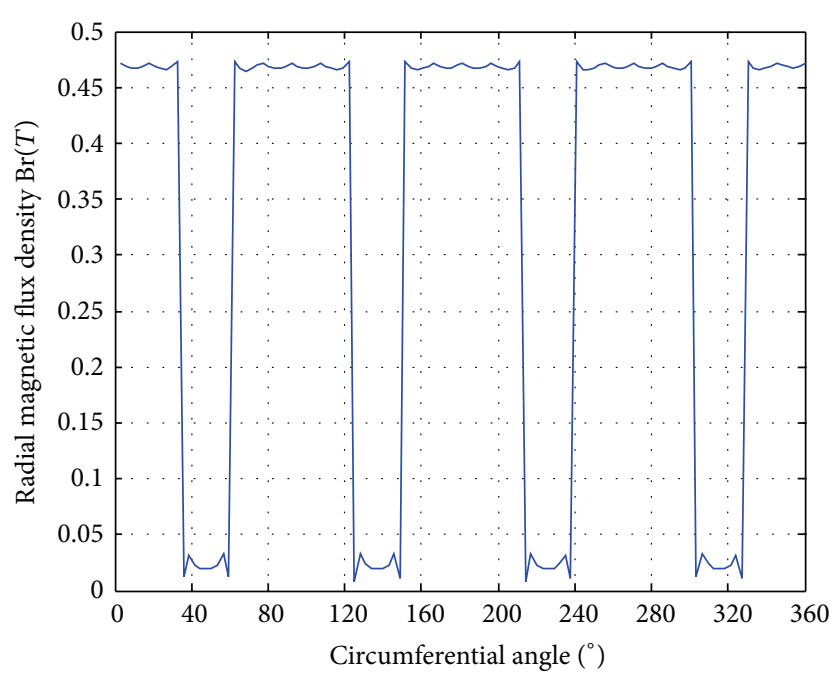

(a)

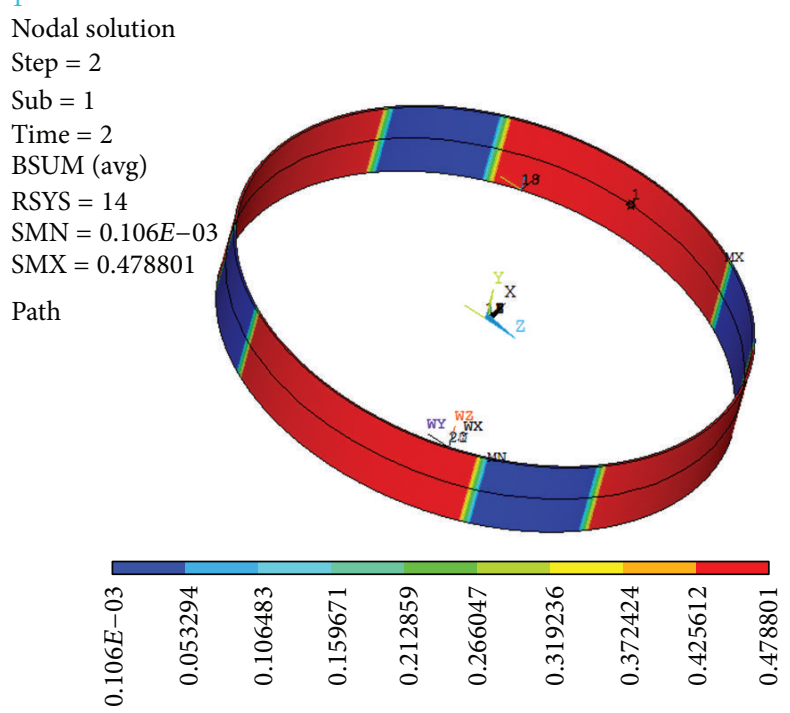

(b)

FIGURE 17: Radial flux density distribution affected only by the permanent magnets in the air gap of RFHMB without pole shoes, (a) radial flux density curves, (b) radial flux density distribution.

both permanent magnets and control current in the air gap of the novel RFHMB without pole shoes are depicted in Figures 17 and 18 . The radial distributions of magnetic density affected by only the permanent magnets or both permanent magnets and control current in the air gap of the novel RFHMB with pole shoes are depicted in Figures 19 and 20.

From Figure 17 to Figure 20, we can find out that due to the existence of pole shoes, the areas of the radial poles for the novel RFHMB are enlarged; then the maximum magnetic density affected by only permanent magnets in the air gap is reduced from $0.47 \mathrm{~T}$ to $0.38 \mathrm{~T}$ while that affected by permanent magnets and control current $0.1 \mathrm{~A}$ in the air gap is reduced from $0.6 \mathrm{~T}$ to $0.52 \mathrm{~T}$; the variations of magnetic density are about $0.08 \mathrm{~T}$; and the reduction of the maximum magnetic density will reduce the eddy loss of the novel RFHMB effectively. On the other hand, we also can find out that due to the existence of pole shoes, the clearance between poles has been reduced from $30^{\circ}$ to $5^{\circ}$, although the fluctuation of the maximum magnetic density affected by only the permanent magnets or both permanent magnets and control current in the air gap still exists but the duty ratio between the maximum magnetic density and minimum magnetic density increases greatly, so the eddy loss of the novel RFHMB will be reduced accordingly. 


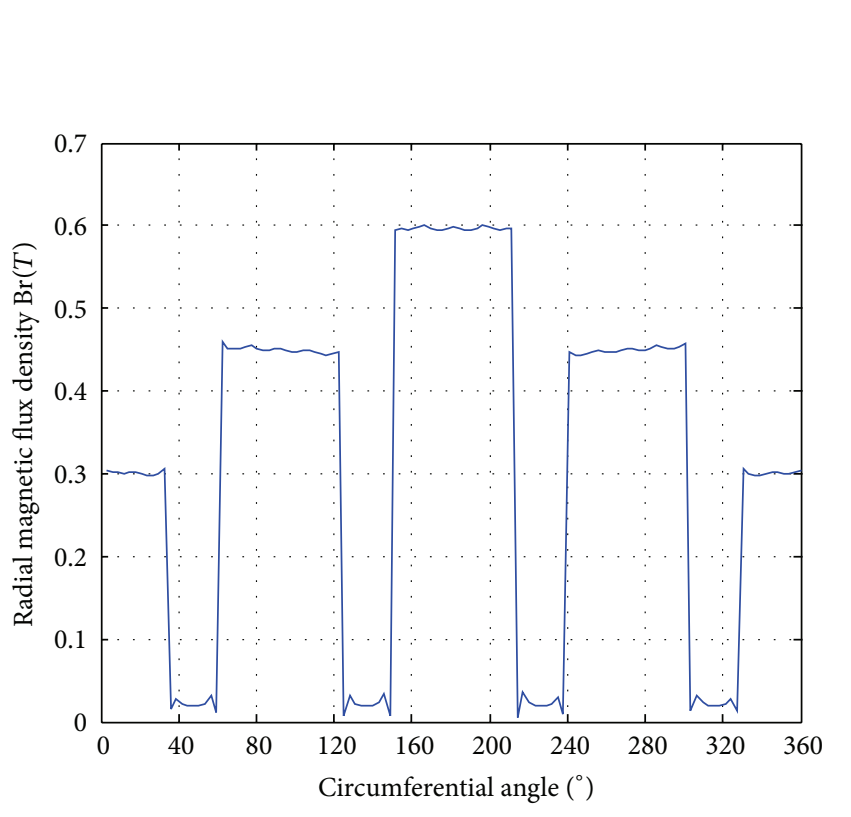

(a)

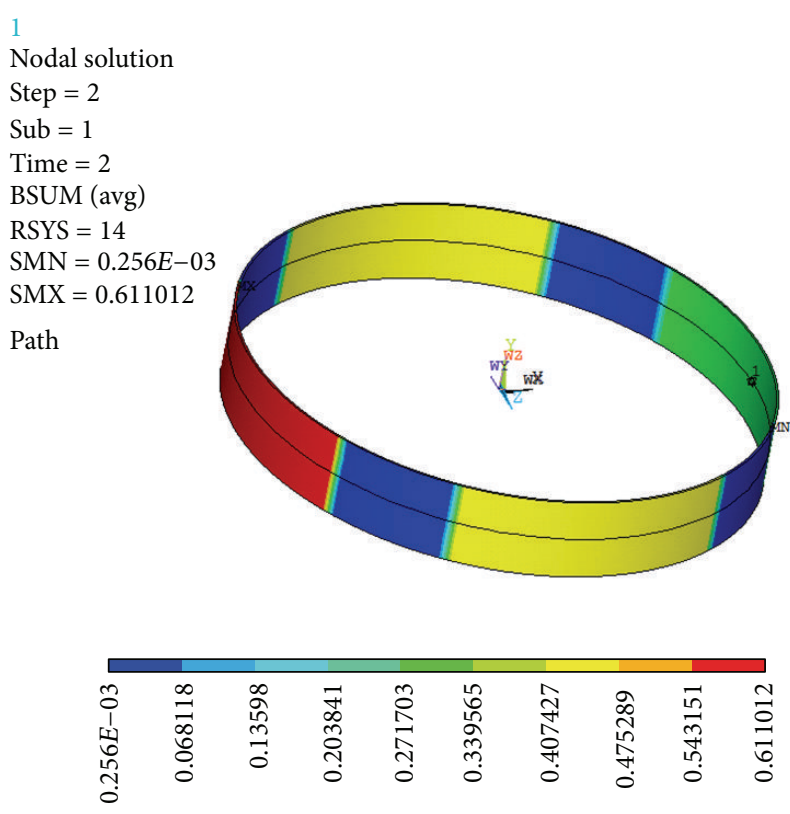

(b)

FIGURE 18: Radial flux density distribution affected by the permanent magnets and the control current $0.1 \mathrm{~A}$ in the air gap of RFHMB without pole shoes, (a) radial flux density curves, (b) radial flux density distribution.

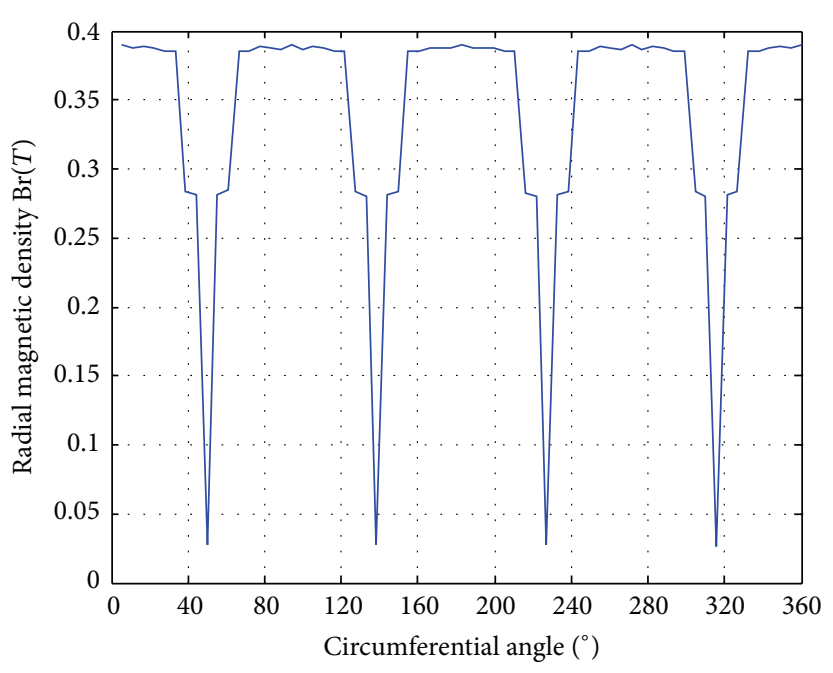

(a)

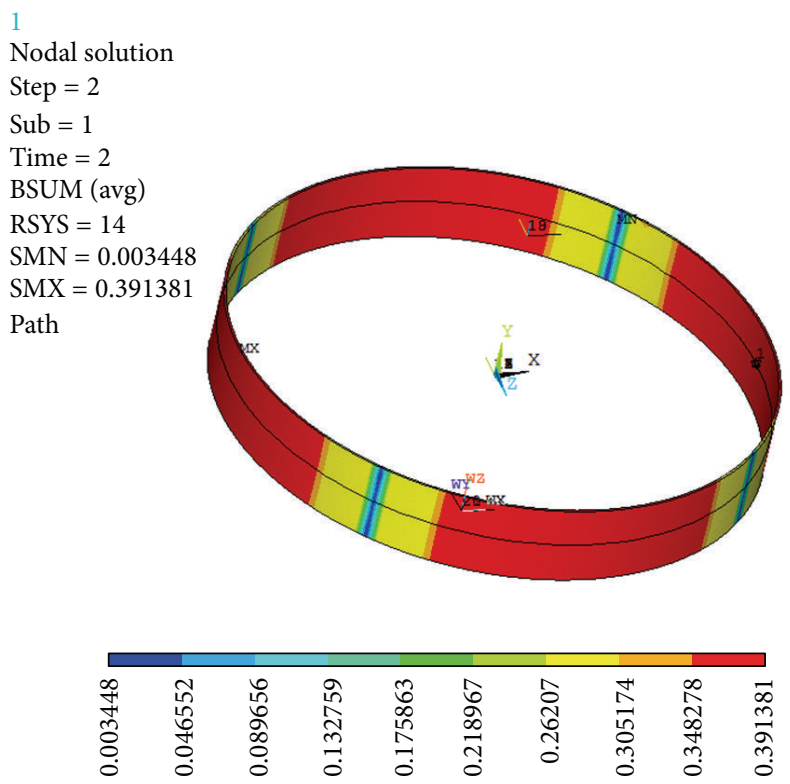

(b)

FIGURE 19: Radial flux density distribution affected by the permanent magnets in the air gap of RFHMB with pole shoes, (a) radial flux density curves, (b) radial flux density distribution.

According to (11), we can find out that the soft magnetic materials with low loss properties such as laminated material, iron-based Nanocrystalline, and amorphousness have great effect on the eddy loss of the novel RFHMB. Some tested results of eddy loss for different soft magnetic materials have verified that the iron loss per unit mass of iron-based Nanocrystalline with $0.03 \mathrm{~mm}$ films is the smallest and the eddy loss of the novel RFHMB is smaller when its rotor made of Nanocrystalline with $0.03 \mathrm{~mm}$ films. Because the iron-based Nanocrystalline with $0.03 \mathrm{~mm}$ films is very crisp and always in reeled state, it is difficult to handle them further, then the iron-based amorphous with $0.1 \mathrm{~mm}$ films is employed for radial rotor of the novel RFHMB.

For a MSFW with polar angular momentum is $68 \mathrm{~N} \cdot \mathrm{m} \cdot \mathrm{s}$ when its rotor rotates at the speed of $5000 \mathrm{r} / \mathrm{min}$, the photo of the novel RFHMB is shown in Figure 21, and its main 


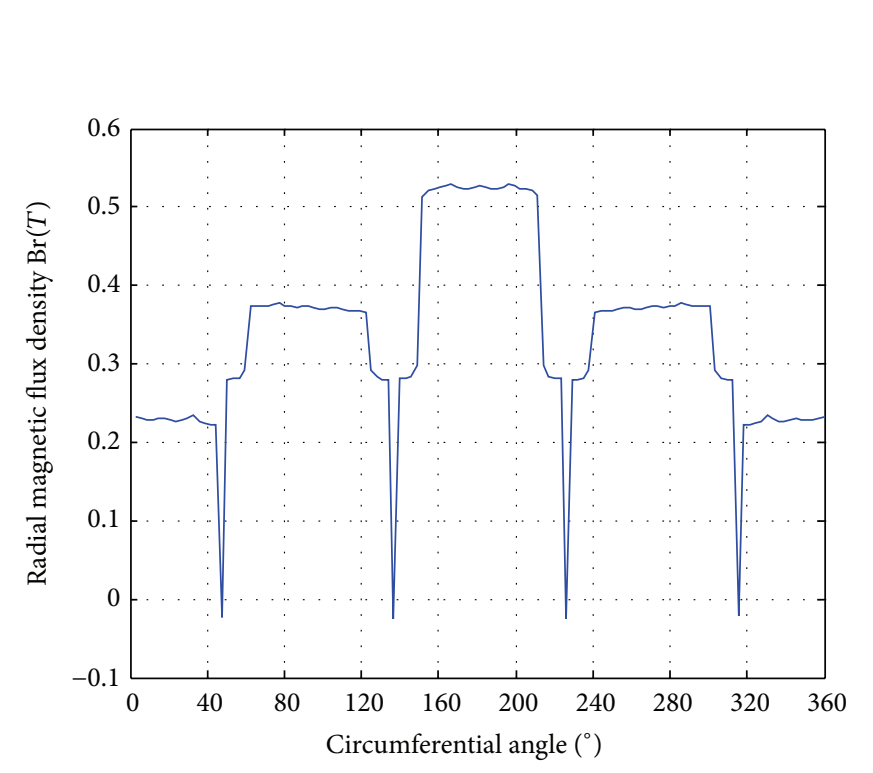

(a)
1

Nodal solution

Step $=2$

$\mathrm{Sub}=1$

Time $=2$

BSUM (avg)

RSYS $=14$

$\mathrm{SMN}=0.003307$

SMX $=0.531598$

Path

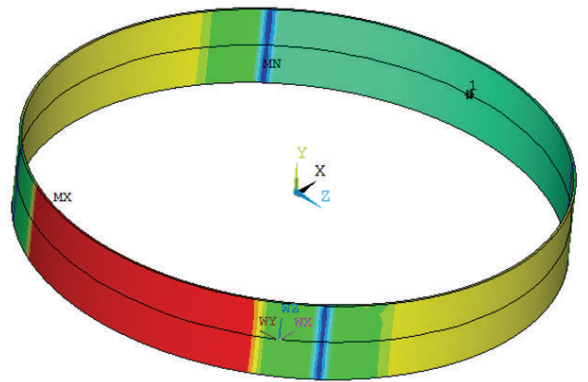

(b)

FIGURE 20: Radial flux density distribution affected by the permanent magnets and the control current $0.1 \mathrm{~A}$ in the air gap of RFHMB with pole shoes, (a) radial flux density curves, (b) radial flux density distribution.

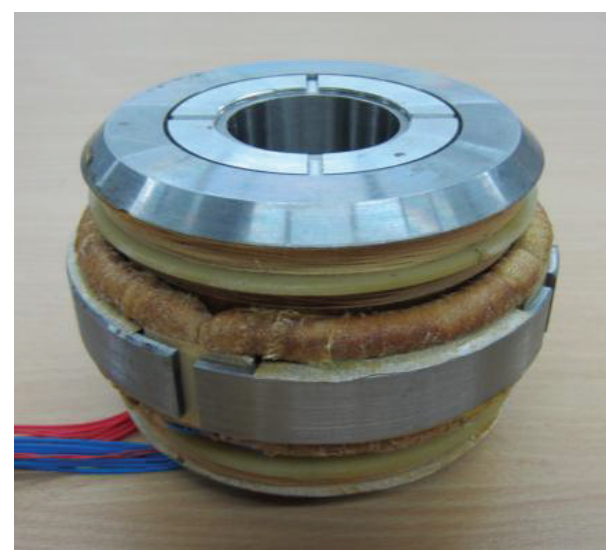

FIGURE 21: Photo of the stator of the novel RFHMB.

parameters are listed in Table 1 . The presented novel RFHMB can make the rotor title around $X$ - or $Y$-axis about $1^{\circ}$ and the generated control moment is up to $3.3 \mathrm{~N} \cdot \mathrm{m}$. It is verified that this novel RFHMB is very helpful to make MSFW with gimballing capability meet the maneuvering requirement of spacecraft fully.

\section{Suspension Properties}

5.1. Noncoupling Suspension Forces. By the principle of virtual work, the component force $F_{r x}$ in $X$ direction and the component force $F_{r y}$ in $Y$ direction of the resultant forces acting on the rotor generated by the radial stators are integrated as

$$
F_{r x}=\int_{\alpha_{11}}^{\alpha_{12}} \frac{\mu_{0} r_{s} h_{r s}\left(\Phi_{x+} R_{x+}\right)^{2} \cdot \cos \theta}{2 \delta_{g}^{2}\left(\delta_{r}, \gamma, \theta\right)} d \theta
$$

$$
\begin{gathered}
+\int_{\alpha_{21}}^{\alpha_{22}} \frac{\mu_{0} r_{s} h_{r s}\left(\Phi_{x-} R_{x-}\right)^{2} \cdot \cos \theta}{2 \delta_{g}^{2}\left(\delta_{r}, \gamma, \theta\right)} d \theta, \\
F_{r y}=\int_{\alpha_{31}}^{\alpha_{32}} \frac{\mu_{0} r_{s} h_{r s}\left(\Phi_{y+} R_{y+}\right)^{2} \cdot \sin \theta}{2 \delta_{g}^{2}\left(\rho_{r}, \gamma, \theta\right)} d \theta \\
+\int_{\alpha_{41}}^{\alpha_{42}} \frac{\mu_{0} r_{s} h_{r s}\left(\Phi_{y-} R_{y-}\right)^{2} \cdot \sin \theta}{2 \delta_{g}^{2}\left(\rho_{r}, \gamma, \theta\right)} d \theta,
\end{gathered}
$$

where $\alpha_{11}, \alpha_{21}, \alpha_{31}$, and $\alpha_{41}$ are the starting angles of the four radial stator poles; $\alpha_{12}, \alpha_{22}, \alpha_{32}$, and $\alpha_{42}$ are the ending angles of the four radial stator poles; $r_{s}$ is the outer radius of the stator; $h_{r s}$ is the height of radial stator iron and $\delta_{g}$ is the length of the radial air gaps, which is variable with the angle $\theta$ around the circumference. $\delta_{r}$ is the rotor displacement in radial direction, $\delta_{r}=\sqrt{\delta_{x}^{2}+\delta_{y}^{2}}, \gamma$ is the direction angle of the rotor displacement vector, and $\gamma=\arctan \left(\delta_{x} / \delta_{y}\right)$.

As for the force generated by the axial conical stator, it includes the component force not only in $Z$ direction but also in $X$ and $Y$ directions. All the component forces $F_{a x}, F_{a y}$ and $F_{a z}$ can be integrated as

$$
\begin{aligned}
F_{a x}= & \int_{0}^{2 \pi} \int_{0}^{L} \frac{\mu_{0}\left(r_{c}-l \cot \varphi\right)\left(\Phi_{\mathrm{au}} R_{\mathrm{au}}\right)^{2} \cdot \cos \theta}{2 \delta_{\mathrm{au}}^{2}\left(\rho_{r}, \gamma, \theta, z\right)} d l d \theta \\
& +\int_{0}^{2 \pi} \int_{0}^{L} \frac{\mu_{0}\left(r_{c}-l \cot \varphi\right)\left(\Phi_{\mathrm{al}} R_{\mathrm{al}}\right)^{2} \cdot \cos \theta}{2 \delta_{\mathrm{al}}^{2}\left(\rho_{r}, \gamma, \theta, z\right)} d l d \theta,
\end{aligned}
$$




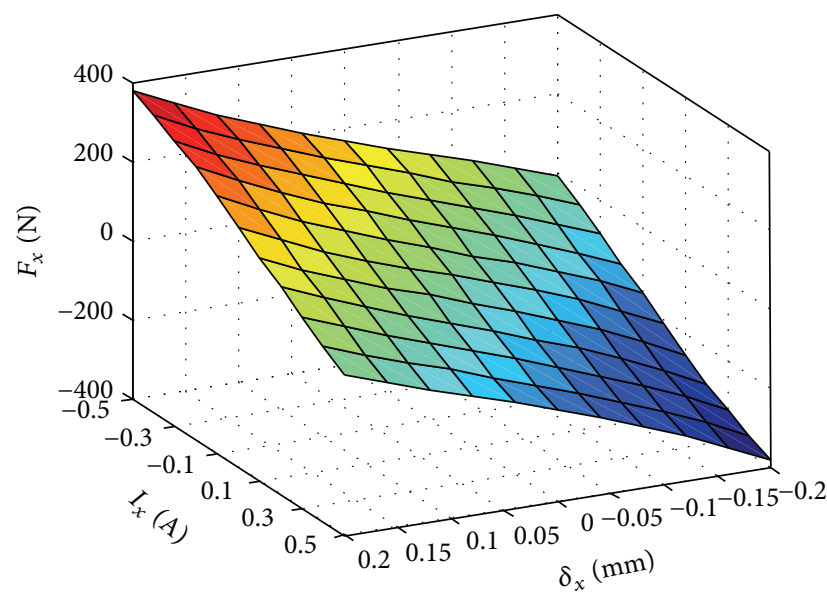

(a)

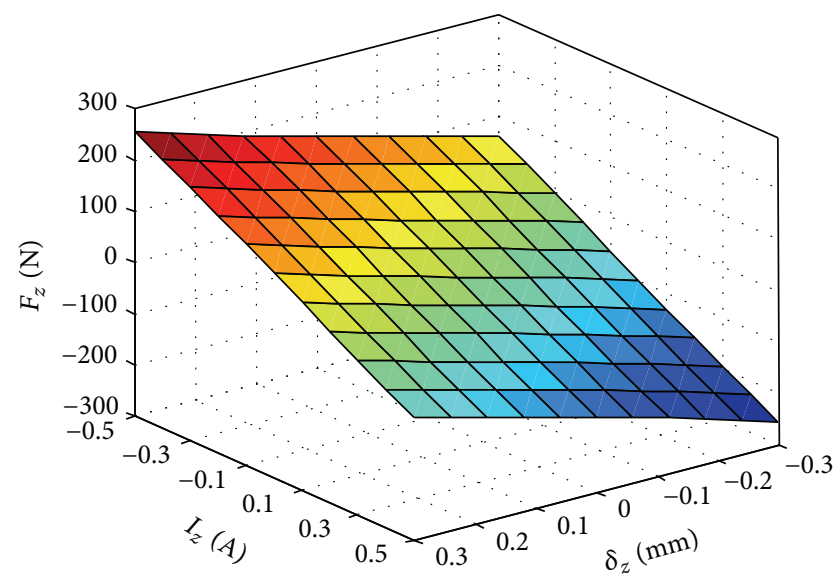

(b)

FIGURE 22: Characteristics of suspension force, (a) $F_{x}$ versus $\delta_{x}$ and $I_{x}$, (b) $F_{z}$ versus $\delta_{z}$ and $I_{z}$.

$$
\begin{aligned}
F_{a y}= & \int_{0}^{2 \pi} \int_{0}^{L} \frac{\mu_{0}\left(r_{c}-l \cot \varphi\right)\left(\Phi_{\mathrm{au}} R_{\mathrm{au}}\right)^{2} \cdot \sin \theta}{2 \delta_{\mathrm{au}}^{2}\left(\rho_{r}, \gamma, \theta, z\right)} d l d \theta \\
& +\int_{0}^{2 \pi} \int_{0}^{L} \frac{\mu_{0}\left(r_{c}-l \cot \varphi\right)\left(\Phi_{\mathrm{al}} R_{\mathrm{al}}\right)^{2} \cdot \sin \theta}{2 \delta_{\mathrm{al}}^{2}\left(\rho_{r}, \gamma, \theta, z\right)} d l d \theta, \\
F_{a z}= & \int_{0}^{2 \pi} \int_{0}^{L} \frac{\mu_{0}\left(r_{c}-l \cot \varphi\right)\left(\Phi_{\mathrm{au}} R_{\mathrm{au}}\right)^{2} \cdot \cos \varphi}{2 \delta_{\mathrm{au}}^{2}\left(\rho_{r}, \gamma, \theta, z\right) \cdot \sin \varphi} d l d \theta \\
& -\int_{0}^{2 \pi} \int_{0}^{L} \frac{\mu_{0}\left(r_{c}-l \cot \varphi\right)\left(\Phi, R_{\mathrm{al}}\right)^{2} \cdot \cos \varphi}{2 \delta_{\mathrm{al}}^{2}\left(\rho_{r}, \gamma, \theta, z\right) \sin \varphi} d l d \theta,
\end{aligned}
$$

where $r_{c}$ is the maximum outer radius of the axial conical stator, $L$ is the axial length of the conical stator iron, and $\delta_{\text {au }}$ and $\delta_{\mathrm{al}}$ are the length of the upper and lower conical air gaps, respectively, both varying with the rotor displacement.

The resultant forces generated by both the radial and the conical stators in $X, Y$, and $Z$ directions can be expressed as

$$
\begin{aligned}
& F_{x}=F_{r x}+F_{a x}, \\
& F_{y}=F_{r y}+F_{a y}, \\
& F_{z}=F_{a z} .
\end{aligned}
$$

Based on the above equations and parameters of the novel RFHMB listed in Table 1, the obtained force-displacement and force-current characteristics are shown in Figure 22. From Figure 22, we can find out that both the radial magnetic force and the axial magnetic force are nearly proportional to the displacement of rotor and their relative control current. We also calculated out that the force-displacement stiffness and force-current stiffness of radial magnetic bearing unit are $0.65 \mathrm{~N} / \mu \mathrm{m}$ and $-380 \mathrm{~N} / \mathrm{A}$, and the force-displacement
TABLE 1: Main parameters of the RFHMB.

\begin{tabular}{lcc}
\hline Symbol & Quantity & $\begin{array}{c}\text { Designed } \\
\text { values }\end{array}$ \\
\hline$\rho_{c 0}$ & Length of conical gap & $0.8 \mathrm{~mm}$ \\
$\rho_{r 0}$ & Length of radial gap & $0.5 \mathrm{~mm}$ \\
$D s_{\mathrm{ra}}$ & Outer diameter of radial stator & $116 \mathrm{~mm}$ \\
$D s_{\mathrm{co}}$ & Maximum outer diameter of conical stator & $102 \mathrm{~mm}$ \\
$L s_{\mathrm{ra}}$ & Height of radial stator iron & $17 \mathrm{~mm}$ \\
$L s_{\mathrm{co}}$ & Height of conical stator & $6.5 \mathrm{~mm}$ \\
$H_{c}$ & Coercive force of permanent magnet & $760 \mathrm{kA} / \mathrm{m}$ \\
$B_{r}$ & Remanence of permanent magnet & $1.05 \mathrm{~T}$ \\
$N_{r}$ & Turns of radial control windings & 220 \\
$N_{a}$ & Turns of axial control windings & 180 \\
$\sigma_{p}$ & Leakage coefficient of bias flux & 1.45 \\
$\sigma_{r}$ & Leakage coefficient of radial control flux & 1.25 \\
$\sigma_{a}$ & Leakage coefficient of axial control flux & 1.2 \\
\hline
\end{tabular}

stiffness and force-current stiffness of axial magnetic bearing unit are $0.34 \mathrm{~N} / \mu \mathrm{m}$ and $-318 \mathrm{~N} / \mathrm{A}$, respectively.

In order to analyze the magnetic coupling among $X$, $Y$, and $Z$ directions, the changes of magnetic forces with variation of current and displacement are analyzed when the rotor is drifted or tilted. Because of the symmetry of the RFHMB structure, not all the force coupling analyses in every direction are presented here. For example, when the rotor is drifted by a certain displacement $\left(\delta_{x}\right)$ in $X$ direction and its corresponding control current $I_{x}$ is given in these windings in this direction, the force in $Y$ direction can be obtained in case that the offset $\delta_{y}=0.1 \mathrm{~mm}$ and the current $I_{y}=0$; the coupling analysis between $X$ and $Y$ channels are illustrated in Figure 23. The relationship among $F_{y}, \delta_{x}$, and $I_{x}$ is shown in Figure 23(a); the relationship among $F_{z}, \delta_{x}$, and $I_{x}$ is shown 

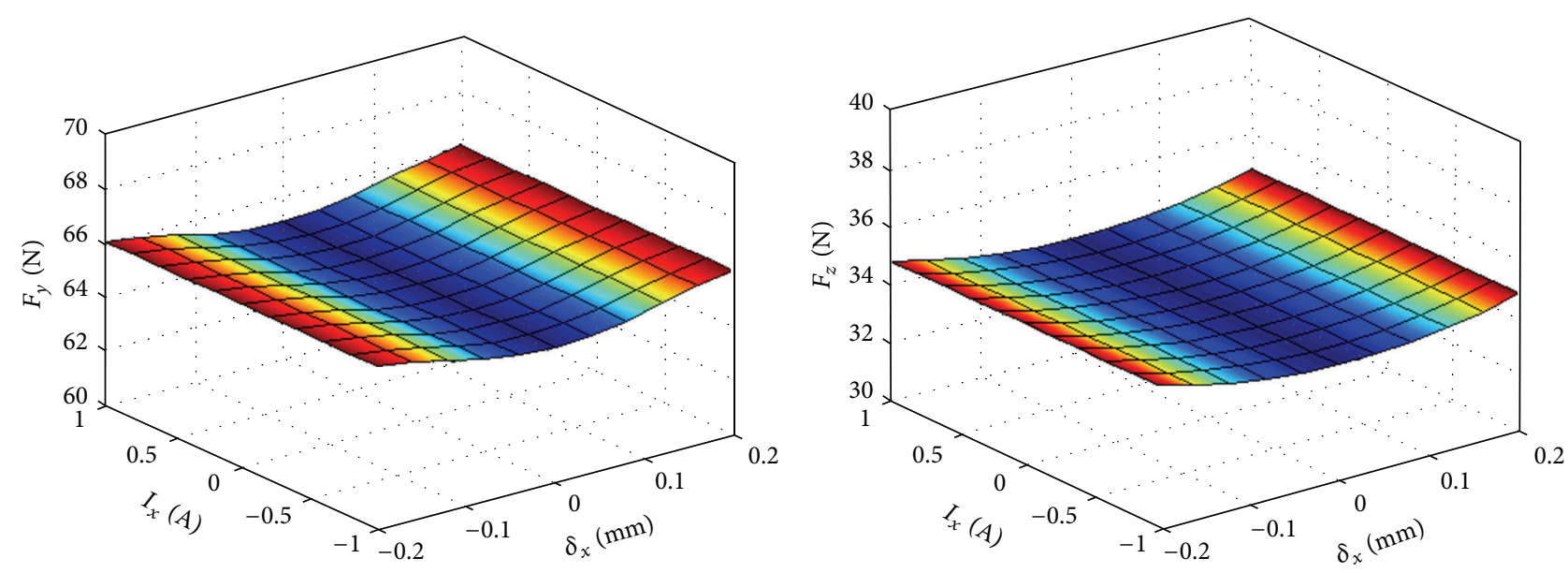

(a)

(b)

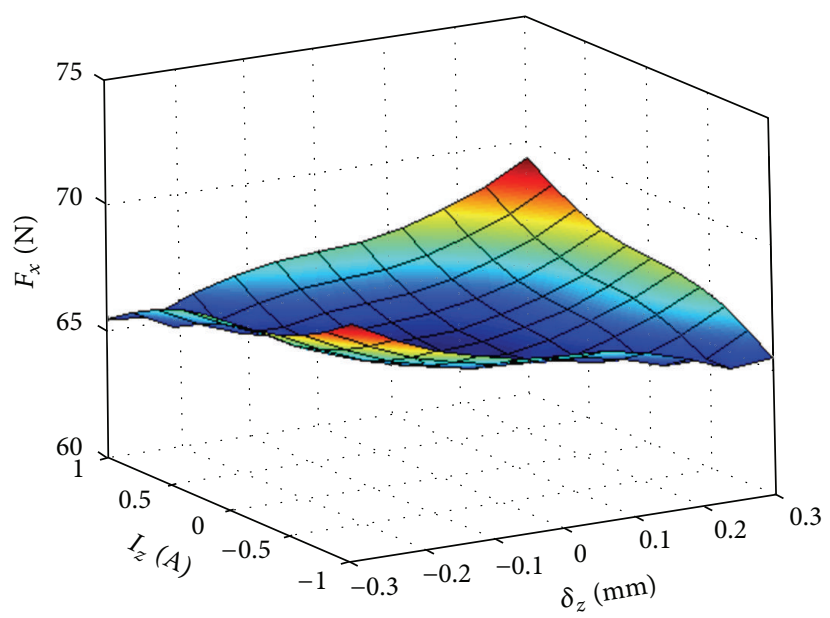

(c)

FIGURE 23: Force coupling among different channels, (a) $F_{y}$ versus $I_{x}$ and $\delta_{x}$, (b) $F_{z}$ versus $I_{x}$ and $\delta_{x}$, (c) $F_{x}$ versus $I_{z}$ and $\delta_{z}$.

in Figure 23(b); and the relationship among $F_{x}, \delta_{z}$, and $I_{z}$ is shown in Figure 23(c), respectively.

We can find out that the offset and control current in windings in one direction have little influence on the force in other directions. When the displacement in $X$ direction reaches $0.25 \mathrm{~mm}$, which is the length of protection air gap, the variation of force in $Y$ direction is only $1.6 \%$ and the variation of force in $Z$ direction is $2.5 \%$, which can be calculated from Figures 23(a) and 23(b). When the position of the rotor is fixed, the forces in $Y$ and $Z$ directions remain unchanged no matter how large the current in $X$ direction is. The force in $X$ direction varies a little with the current and displacement in $Z$ direction due to the radial component force generated by the conical stator, and its maximum variation is not better than $6 \%$. Therefore, magnetic fluxes of the novel RFHMB are almost decoupled among different directions when the rotor shifts in some direction.
When the rotor tilts around $X$ - or $Y$-axis, the force coupling is also analyzed in case that the rotor drifts a certain displacement in one direction, that is, $X, Y$, or $Z$ direction. These main analysis results are illustrated in Figure 24 to express the relationships between $F_{z}$ and $\alpha, F_{x}$ and $\alpha$, and $F_{y}$ and $\alpha$, respectively.

We can find out that when the centroid of the rotor coincides with that of the novel RFHMB, that is, the rotor does not drift in any direction ( $X, Y$, or $Z$ direction), the rotor tilting has no influence on its relative force. But when the rotor drifts in some direction, the force in this direction will increase with the increasing of the tilting angle whether the tilting angle is positive or negative. When the rotor tilts around $X$-axis up to the designed maximum angle $1^{\circ}$, the force change in $Z$ direction is $9 \%$ in case of a axial displacement $0.3 \mathrm{~mm}$ while the force change will reach $3 \%$ in $X$ directions or $14 \%$ in $Y$ directions in case of a 


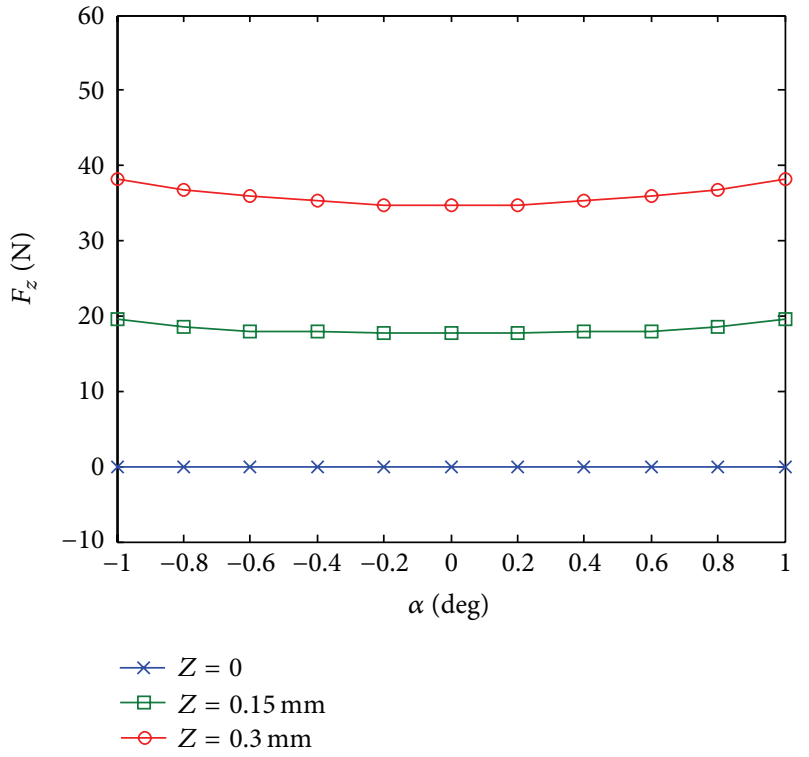

(a)

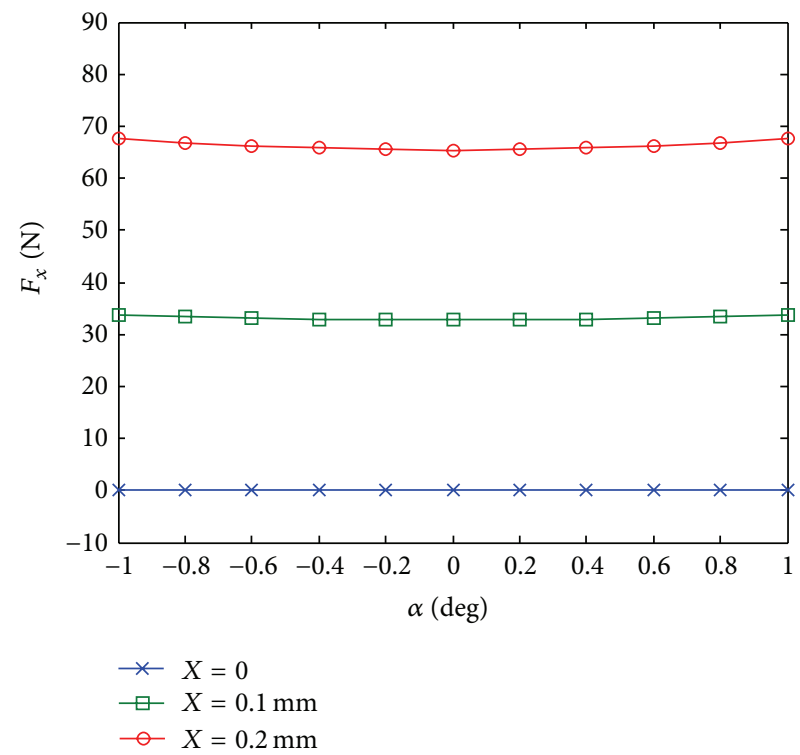

(b)

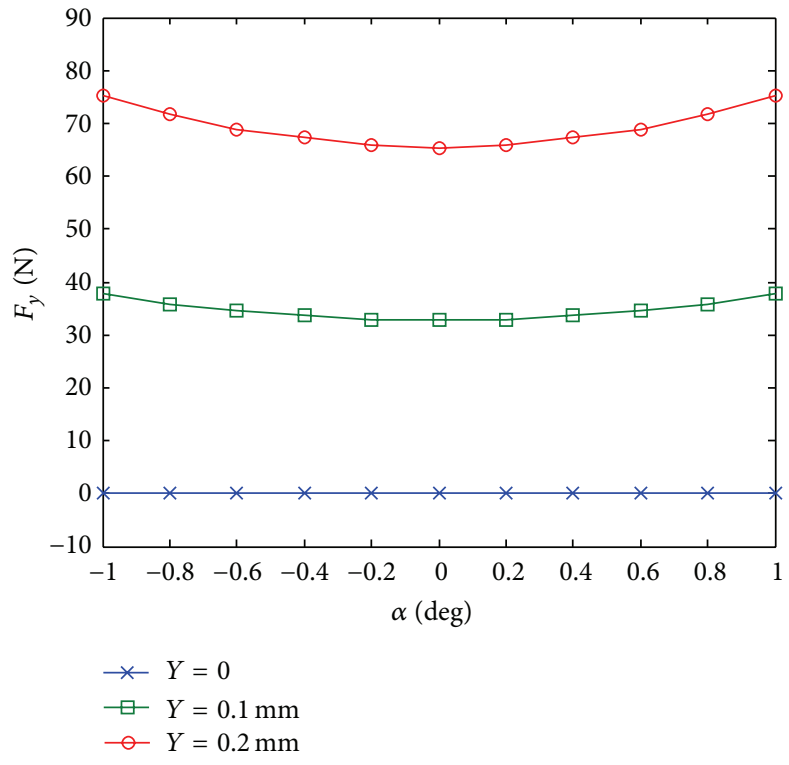

(c)

FIGURE 24: Force coupling when rotor tilts, (a) $F_{z}$ versus $\alpha$, (b) $F_{x}$ versus $\alpha$, (c) $F_{y}$ versus $\alpha$.

radial displacement $0.2 \mathrm{~mm}$, respectively. Therefore, the rotor should be well suspended in the central position before the rotor is tilted to generate the required control moment.

5.2. Low Additional Tilting Torque. When the rotor tilts around $X$ - or $Y$-axis, the additional tilting torque is caused mainly by the conical stators, especially when the normal direction of the conical faces departs from the center of the rotor. Assuming the rotor tilts around $X$ - and $Y$-axes by the angles of $\alpha$ and $\beta$, respectively, the torque can be derived as

$$
M_{x}=\int_{0}^{2 \pi} \int_{0}^{L} \frac{\mu_{0}\left(r_{c}-l \cot \varphi\right)\left(\phi_{\mathrm{au}} R_{\mathrm{au}}\right)^{2}}{2 \delta_{\mathrm{au}}^{2}(\theta, \alpha, \beta) \sin \varphi} \cdot l_{x}(\theta, l) d \theta d l
$$

$$
\begin{aligned}
& -\int_{0}^{2 \pi} \int_{0}^{L} \frac{\mu_{0}\left(r_{c}-l \cot \varphi\right)\left(\phi_{\mathrm{au}} R_{\mathrm{au}}\right)^{2}}{2 \delta_{\mathrm{al}}^{2}(\theta, \alpha, \beta) \sin \varphi} \cdot l_{x}(\theta, l) d \theta d l, \\
M_{y}= & \int_{0}^{2 \pi} \int_{0}^{L} \frac{\mu_{0}\left(r_{c}-l \cot \varphi\right)\left(\Phi_{\mathrm{au}} R_{\mathrm{au}}\right)^{2}}{2 \delta_{\mathrm{au}}^{2}(\theta, \alpha, \beta) \sin \varphi} \cdot l_{y}(\theta, l) d \theta d l \\
& -\int_{0}^{2 \pi} \int_{0}^{L} \frac{\mu_{0}\left(r_{c}-l \cot \varphi\right)\left(\Phi_{\mathrm{au}} R_{\mathrm{au}}\right)^{2}}{2 \delta_{\mathrm{al}}^{2}(\theta, \alpha, \beta) \sin \varphi} \cdot l_{y}(\theta, l) d \theta d l,
\end{aligned}
$$

where $l_{x}(\theta, l)=\left(L_{p}+l\right) \sin \varphi \cdot \sin \theta-\left(r_{c}-l \cot \varphi\right) \cos \varphi \cdot \sin \theta$ and $l_{y}(\theta, l)=\left(L_{p}+l\right) \sin \varphi \cdot \cos \theta-\left(r_{c}-l \cot \varphi\right) \cos \varphi \cdot \cos \theta$. 


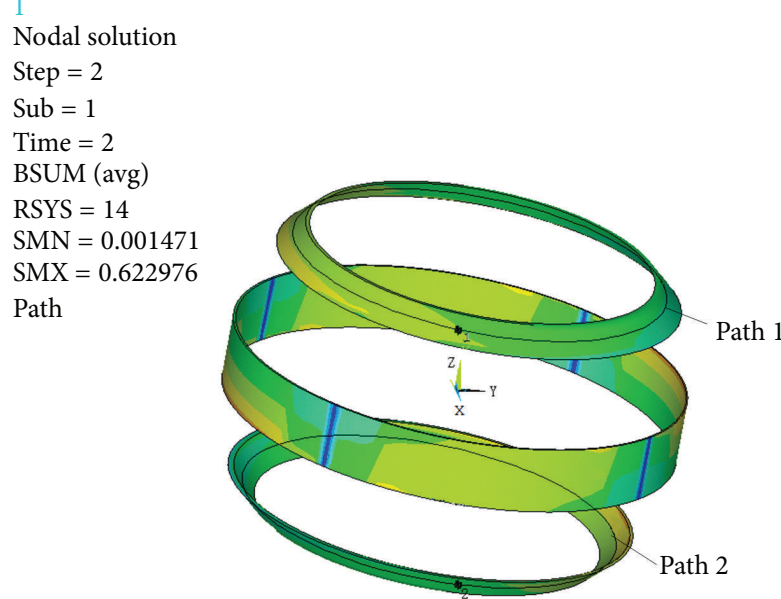

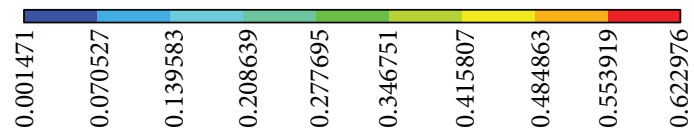

(a)

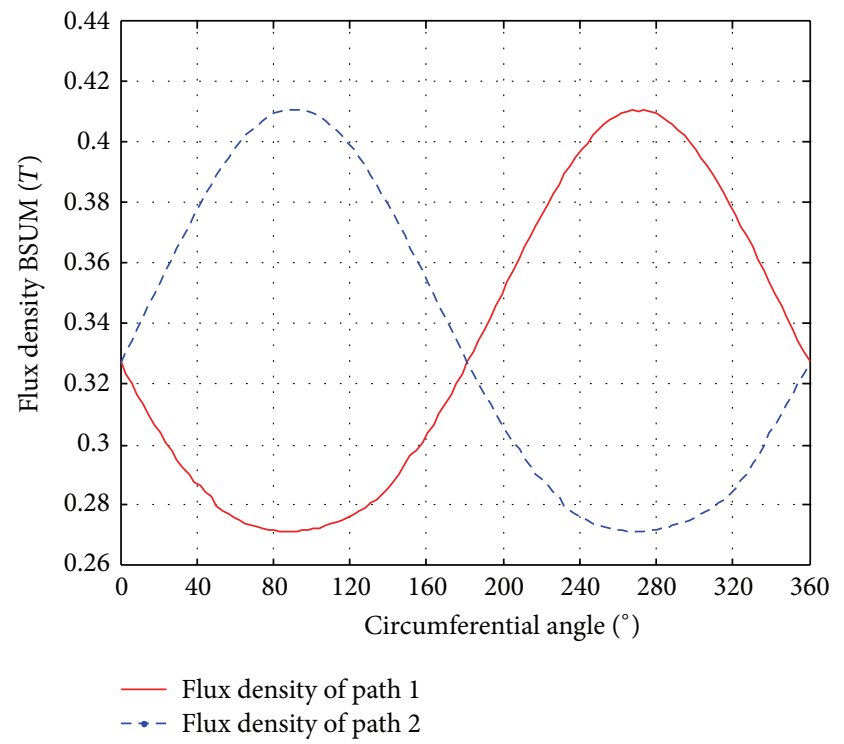

(b)

Figure 25: Magnetic density fluctuation in the air gap of RFHMB (a) Magnetic field distribution (b) Magnetic flux density curves along path 1 and 2.

When the rotor is tilted around $X$ - or $Y$-axes, the additional tilting torque is mainly caused by the unbalanced force and its related torque arm. The unbalanced force is determined by the uniform distribution of the flux density around the circumference. In case that the rotor tilts around $X$-axis about $1^{\circ}$, the distributions of flux density in the radial and axial air gaps derived by the finite element method (FEM) are shown in Figure 25(a). The corresponding flux density curves along path 1 and path 2 are shown in Figure 25(b).

From Figure 25, we can find out that when the rotor tilts around $X$ - or $Y$-axis, the flux density of the conical air gap is uniform around the circumference. The fluctuation of the flux density is from $0.272 \mathrm{~T}$ to $0.41 \mathrm{~T}$. The variation trend of the flux density in upper and lower conical air gaps is opposite at any circumferential angle. Therefore, the related magnetic force is uniform around the circumference and the forces of upper and lower conical rotors cannot be counteracted at the same circumferential angle. With respect to the conical rotors, only the normal direction of the conical midst faces directs to the centroid of the rotor, so the torque arm still exists and an additional torque around $X$ - or $Y$ axis is generated unavoidably. For the conical axial magnetic bearing in the presented novel RFHMB, the additional torque $M_{\mathrm{a}-\mathrm{r}}$ can be calculated as

$$
M_{\mathrm{a}-\mathrm{r}}=\int_{S 1} \frac{b_{1}^{2}(\theta) \cdot l}{2 \mu_{0}} d A+\int_{S 2} \frac{b_{2}^{2}(\theta) \cdot l}{2 \mu_{0}} d A,
$$

where $b_{1}(\theta)$ and $b_{2}(\theta)$ are the flux densities that vary with the circumferential angle $\theta ; S 1$ and $S 2$ are the areas of upper and lower conical stators, respectively. $l$ is the normal distance between the vector of the unbalanced force generated by the conical axial magnetic bearing and the centroid of the rotor; it is very small and can be neglected in most cases.
On the other hand, if the rotor of the MSFW with gimballing capability is suspended by the common reluctance force-type magnetic bearing as shown in Figure 5, the generation of additional tilting torque on rotor is shown in Figure 8. Because the working surface of the 3-DOF MB is plane faces and the torque arm is comparatively large, its additional torque is expressed by (6). Considering the uniform distribution of the flux density and the additional torque generated by the radial rotors, the relationships between the tilting angle $\alpha$ and the additional tilting torque $M$ of the novel RFHMB and that of the common reluctance force-type magnetic bearing with plane faces are analyzed by FEM and these results are illustrated in Figure 26.

From Figure 26, we can find out that the additional tilting torque for the presented novel RFHMB is $0.05 \mathrm{Nm}$, which is very small, but the additional tilting torque for the common reluctance force-type magnetic bearing increases with the angle linearly and its maximum torque is up to $11 \mathrm{Nm}$. We also find out that the angular stiffness calculated from its torque-tilting angle curve for the presented novel RFHMB is $0.11 \mathrm{Nm} /{ }^{\circ}$ while the calculated angular stiffness of the common reluctance force-type magnetic bearing is as much as $10.83 \mathrm{Nm} /{ }^{\circ}$. So the presented novel RFHMB with low additional tilting torque will make the MSFW with gimballing capability have high-precision attitude control ability for spacecraft.

\section{Conclusion}

With respect to this MSFW with gimballing capability, magnetic bearings can be divided into Lorentz force-type magnetic bearings and reluctance force-type magnetic bearings according to the physical cause of the generation of 


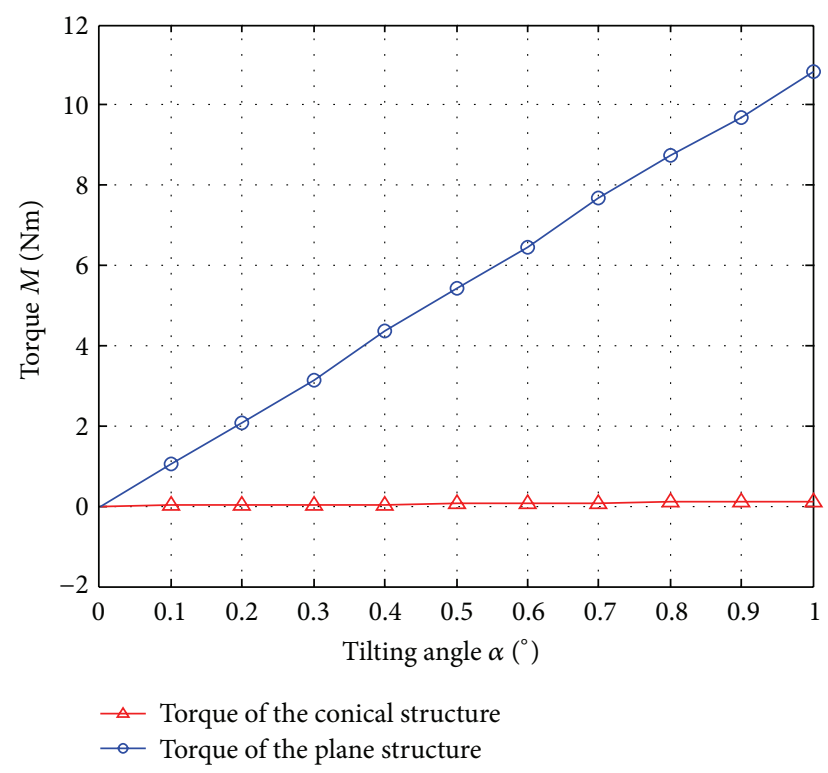

FIGURE 26: Torque characteristics of the presented conical RFHMB and the comparative plane structure.

the force. The demerits of common reluctance force-type magnetic bearing for flywheel with gimballing capability are the existence of positive displacement stiffness, which will result in unbalanced forces when the rotor tilts. For the novel RFHMB, its novelties are the radial and axial magnetic bearing units which are integrated as a compact assembly, permanent magnets are used to generate the radial and axial biased fluxes, the radial stators include 4 stator poles, and their relative permanent magnets are also separated into four separate segments to make the magnetic flux of $X$ and $Y$ channels independent from each other. Two conical stators are used to control the translation of rotor in only $Z$ direction without having effects on these forces in radial direction and to make the additional torque acting on the rotor zero approximately when the rotor is tilted.

The equivalent magnetic circuit of permanent magnets and the equivalent magnetic circuits of control current are researched and their main parameters obtained. To reduce the eddy loss caused unavoidably by the segmented radial stator poles, the radial stator poles are designed as four separate poles with shoes and their rotor is made of ironbased amorphous. When the rotor tilts around $X$ - or $Y$ axis, simulation results indicated that the fluctuation of flux density is from $0.272 \mathrm{~T}$ to $0.41 \mathrm{~T}$, the additional tilting torques in the novel RFHMB is $0.05 \mathrm{Nm}$ while that in the common reluctance force-type magnetic bearing increases with the angle linearly and its maximum torque is up to $11 \mathrm{Nm}$. By analyzing the changes of magnetic forces with variation of current and displacement, we found out that magnetic fluxes of the novel RFHMB are almost decoupled among different directions when the rotor drifts in some direction. When the rotor tilts around $X$ - or $Y$-axis, there are 2 conclusions: (1) the rotor tilting has no influence on its relative force if the centroid of the rotor coincides with that of the novel RFHMB; (2) the force in this direction will increase with the enlargement of the tilting angle whether the tilting angle is positive or negative when the rotor drifts in some direction, but the maximum changes do not exceed $14 \%$. This analysis proved that not only the magnetic fluxes of the radial magnetic bearing unit in $X$ and $Y$ channels are independent from each other, but also the magnetic fluxes of these conical stators have no effect on that of the radial magnetic bearing unit. All these results proved that the gimballing MSFW with the novel RFHMB can meet the maneuvering requirement of spacecraft theoretically.

\section{Acknowledgment}

This work was supported by the National Natural Science Foundation of China under Grant 61174003.

\section{References}

[1] B. Penné, C. Tobehn, M. Kassebom, and B. Ziegler, "A high agile satellite PLATFO50 FO5 EA5 th OB6E5 vation -performance description using new generation missions," in Proceedings of the AIAA 57th International Astronautical Congress (IAC '06), pp. 3262-3272, Valencia, Spain, October 2006.

[2] P. Butz and U. Renner, "A mocrosat-bus for earth observation payloads," in Proceedings of the the 3rd International Symposium on Small Satellites Systems and Services, Annecy, France, 1996.

[3] P. Silvestrin, "Control and navigation aspects of the new Earth observation missions of the European Space Agency," Annual Reviews in Control, vol. 29, no. 2, pp. 247-260, 2005.

[4] V. Lappas, D. Richie, C. Hall, J. Fausz, and B. Wilson, "Survey of technology developments in flywheel attitude control and energy storage systems," Journal of Guidance, Control, and Dynamics, vol. 32, no. 2, pp. 354-365, 2009.

[5] A. Nakajima and C. Murakami, "Active nutation control of spacecraft using a magnetic bearing momentum wheel with vernier gimballing capability," Tech. Rep. TR-820T, National Aerospace Laboratory, 1984.

[6] T. Hashimoto, T. Hamasaki, I. Nakatani, and K. Ninomiya, "Attitude control system design of a satellite with a magnetically suspended momentum wheel based on two-degree-of-freedom control system theory," AIAA-93-3844-CP, 1993.

[7] Y. C. Xie, H. Sawada, and T. Hashimoto, "Actively controlled magnetic bearing momentum wheel and its application to satellite attitude control," The Institute of Space and Astronautical Science Report 680, 2001.

[8] J. G. Bitterly, "Flywheel technology: past, present, and 21st century projections," IEEE Aerospace and Electronic Systems Magazine, vol. 13, no. 8, pp. 13-16, 1998.

[9] K. R. Rajagopal and K. K. Sivadasan, "Low-stiction magnetic bearing for satellite application," Journal of Applied Physics, vol. 91, no. 10, pp. 6994-6996, 2002.

[10] J. C. Fang and Y. Ren, "High-precision control for a singlegimbal magnetically suspended control moment gyro based on inverse system method," IEEE Transactions on Industrial Electronics, vol. 58, pp. 4331-4342, 2011.

[11] T. Mizuno, "Analysis on the fundamental properties of active magnetic bearing control systems by a transfer function approach," JSME International Journal C, vol. 44, no. 2, pp. 367373, 2001.

[12] B. Gerlach, M. Ehinger, H. K. Raue, and R. Seiler, "Digital controller for a gimballing magnetic bearing reaction wheel," 
in Proceedings of the AIAA Guidance, Navigation, and Control Conference, pp. 6244-6249, San Francisco, Calif, USA, August 2005.

[13] H. Sawada, T. Hashimoto, and K. Ninomiya, "High-stability attitude control of satellites by magnetic bearing wheels," in Proceedings of the AIAA Guidance, Navigation, and Control Conference and Exhibit, Denver, Colo, USA, August 2000.

[14] Y. Kim, R. Beach, A. Palazzolo, and A. Provenza, "Interactions dynamics between a satellite and onboard magnetically suspended flywheels," in Proceedings of the 1st International Energy Conversion Engineering Conference, Portsmouth, Va, USA, 2003.

[15] U. Bichler and T. Eckardt, "A 3(5) degree of freedom electrodynamic bearing wheel for 3 -axis spacecraft attitude control applications," in Proceedings of the 1st International Symposium on Magnetic Bearings, pp. 13-22, ETH Zurich, Switzerland, June 1988.

[16] K. Yabuuchi, M. Inoue, and S. Akishita, "A compact magnetic bearing for gimbaled momentum wheel," in Proceedings of the 17th Aerospace Mechanisms Symposium, Pasadena, Calif, USA, 1983.

[17] H. Y. Kim and C. W. Lee, "Design and control of active magnetic bearing system with Lorentz force-type axial actuator," Mechatronics, vol. 16, no. 1, pp. 13-20, 2006.

[18] T. Eckardt, “The low noise momentum wheel MWX EM design and predicted properties," in Proceedings of the 5th European Space Mechanisms and Tribology Symposium, Noordwijk, The Netherlands, 1992.

[19] J. C. Fang, J. J. Sun, H. Liu, and J. Q. Tang, "A novel 3-DOF axial hybrid magnetic bearing," IEEE Transactions on Magnetics, vol. 46, no. 12, pp. 4034-4045, 2010.

[20] J. J. Sun and J. C. Fang, "A novel structure of permanent-magnetbiased radial hybrid magnetic bearing," Journal of Magnetism and Magnetic Materials, vol. 323, no. 2, pp. 202-208, 2011.

[21] J. Q. Tang, J. J. Sun, J. C. Fang, and S. Z. Sam Ge, "Low eddy loss axial hybrid magnetic bearing with gimballing control ability for momentum flywheel," Journal of Magnetism and Magnetic Materials, vol. 329, pp. 153-164, 2013.

[22] Q. Wu, W. Pan, Y. Hong, Y. Sun, and H. Zhu, "Principle and parameter design for an innovated radial-axial hybrid magnetic bearing," in Proceedings of the 11th International Conference on Electrical Machines and Systems (ICEMS '08), pp. 2142-2146, October 2008.

[23] Y. Horiuchi and M. Inoue, "Development of magnetic bearing momentum wheel for ultra-precision spacecraft attitude control," in Proceedings of the 7th International Symposium on Magnetic Bearings, ETH, Zurich, Switzerland, 2000.

[24] J. Watkins, G. Brown, and K. Blumenstock, "Control of integrated radial and axial magnetic bearings," in Proceedings of the 33rd Southeastern Symposium on System Theory, Athens, Ga, USA, March 2001. 


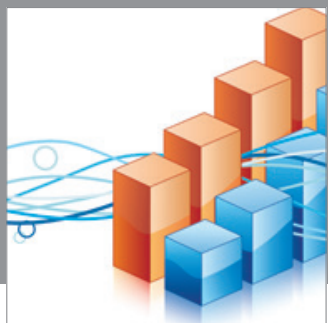

Advances in

Operations Research

mansans

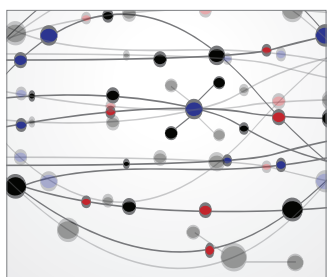

The Scientific World Journal
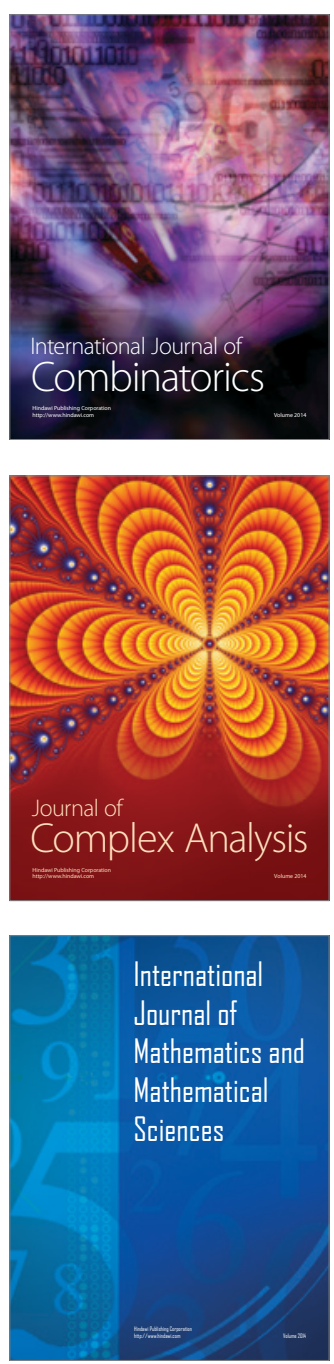
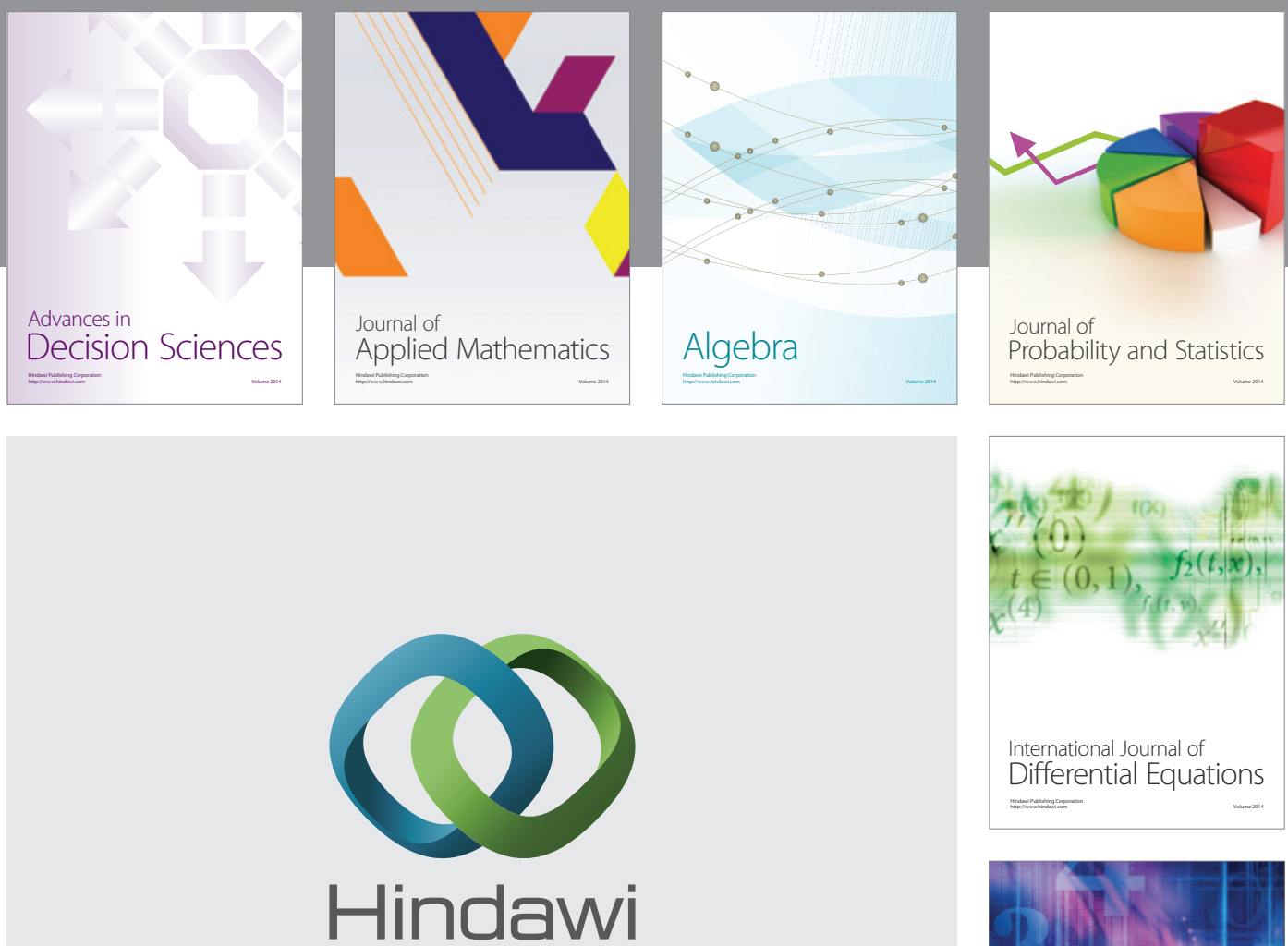

Submit your manuscripts at http://www.hindawi.com
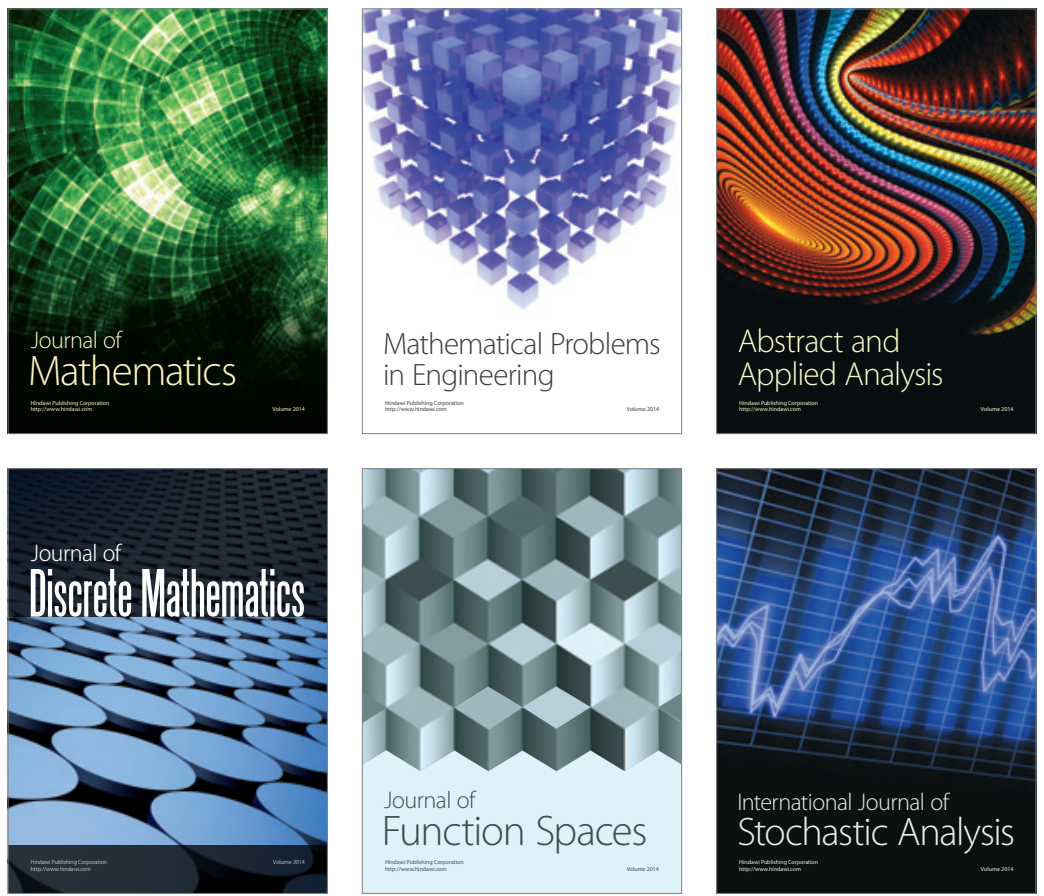

Journal of

Function Spaces

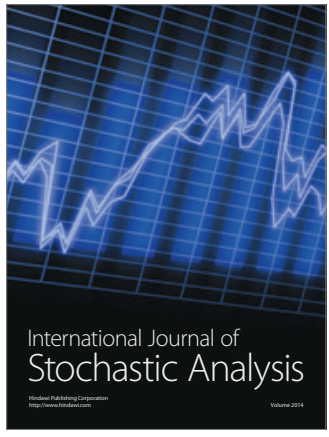

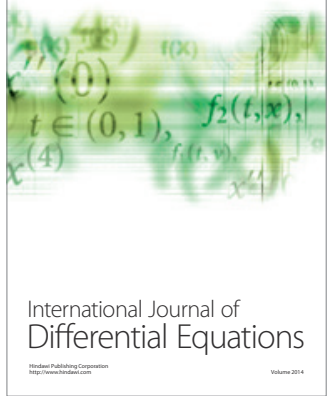
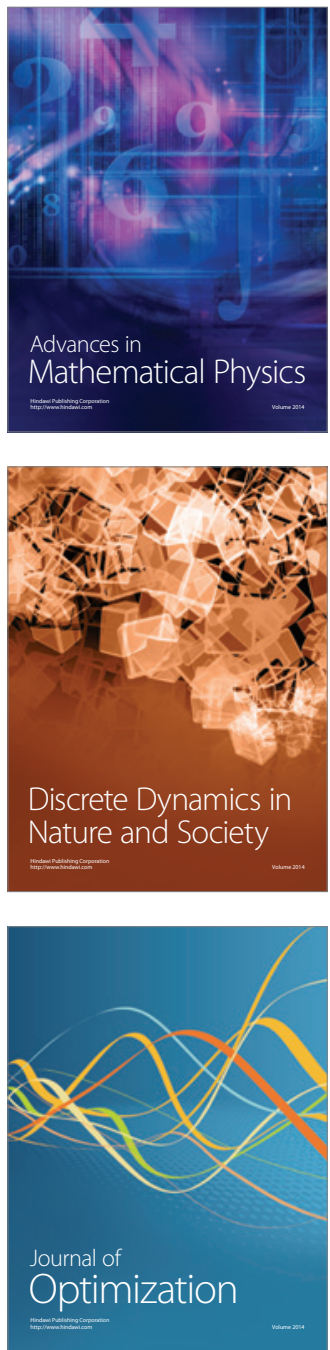